\title{
SUBSEQUENTIAL MINIMALITY IN GOWERS AND MAUREY SPACES
}

\author{
VALENTIN FERENCZI AND THOMAS SCHLUMPRECHT
}

\begin{abstract}
We define block sequences $\left(x_{n}\right)$ in every block subspace of a variant of the space of Gowers and Maurey so that the map $x_{2 n-1} \mapsto x_{2 n}$ extends to an isomorphism. This implies the existence of a subsequentially minimal HI space, which solves a question in [FR1].
\end{abstract}

\section{Contents}

1. Introduction

1.1. Gowers' classification program

1.2. Ranges and supports

1.3. The list of 6 inevitable classes

1.4. The main result

1.5. Some comments on our construction

1.6. Some preliminary definitions

2. The space $S$

2.1. Upper bounds of $\|\cdot\|_{S}$

2.2. Yardstick vectors

3. Construction of a version of Gowers Maurey space

4. Some technical observations concerning the space $\mathcal{G M}$

5. Yardstick Vectors in $\mathcal{G M}$

6. Construction of two equivalent intertwined sequences

7. Consequences of the main result

7.1. Asymptotic unconditionality

7.2. Applications of Gowers' Theorem

7.3. Local minimality

7.4. Open problems

References

2000 Mathematics Subject Classification. 46B03, 03E15.

The second author's research is partially supported by NSF grant DMS0856148. A large part of the research for this paper was accomplished during a visit of the second author to the Universidade de São Paulo which was funded by FAPESP grant 2010/17493-1. 


\section{INTRODUCTION}

We start this article by motivating our result with a presentation of W.T. Gowers's program of classification of Banach spaces, and its recent developments along the lines of [FR1, FR2] and [FG].

\subsection{Gowers' classification program.}

W.T. Gowers' fundamental results in geometry of Banach spaces [G3, G4] opened the way to a loose classification of Banach spaces up to subspaces, known as Gowers' program. The aim of this program is to produce a list of classes of infinite dimensional Banach spaces such that:

(a) the classes are hereditary, i.e., stable under taking subspaces (or block subspaces),

(b) the classes are inevitable, i.e., every infinite dimensional Banach space contains a subspace in one of the classes,

(c) the classes are mutually disjoint,

(d) belonging to one class gives some information about the operators that may be defined on the space or on its subspaces.

We shall refer to such a list as a list of inevitable classes of Gowers. The reader interested in more details about Gowers' program may consult [G4] and [FR1]. Let us just say that the class of spaces $c_{0}$ and $\ell_{p}$ is seen as the most regular class, and so, the objective this program really is the classification of those spaces which do not contain a copy of $c_{0}$ or $\ell_{p}$. We shall first give a summary of the classification obtained in [FR1] and of the results of Gowers that led to it.

The first classification result of Gowers was motivated by his construction with B. Maurey of a hereditarily indecomposable (or HI) space $G M$, i.e., a space such that no subspace may be written as the direct sum of infinite dimensional subspaces [GM]. The space $G M$ was the first known example of a space without an unconditional sequence. Gowers then proved his first dichotomy.

Theorem 1.1 (First dichotomy [G3]). Every Banach space contains either an HI subspace or a subspace with an unconditional basis.

These were the first two examples of inevitable classes.

After $G M$ was defined, Gowers was able to apply a criterion of P.G. Casazza to prove that an unconditional Gowers-Maurey's space $G_{u}$ is isomorphic to no proper subspace, solving Banach's hyperplane problem [G1]. Later on Gowers and Maurey proved that $G M$ also solves Banach's hyperplane problem, but as a consequence of general properties of HI spaces, based on Fredholm theory, rather than by applying the criterion. Let us note in passing that our main result will suggest that Casazza's criterion is indeed not satisfied in Gowers-Maurey's space.

Gowers then refined the list by proving a second dichotomy as a consequence of his general Ramsey theorem for block sequences [G4]. A space is said to be quasi-minimal if any two subspaces have further subspaces which are isomorphic. 
Theorem 1.2 (Second dichotomy G4]). Every Banach space contains a quasi-minimal subspace or a subspace with a basis such that no two disjointly supported block subspaces are isomorphic.

Finally, H. Rosenthal had defined a space to be minimal if it embeds into any of its subspaces. A quasi minimal space which does not contain a minimal subspace is called strictly quasi minimal, so Gowers again divided the class of quasi minimal spaces into the class of strictly quasi minimal spaces and the class of minimal spaces.

Gowers deduced from these dichotomies a list of four inevitable classes of Banach spaces: HI spaces, such as $G M$; spaces with bases such that no disjointly supported subspaces are isomorphic, such as $G_{u}$; strictly quasi minimal spaces with an unconditional basis, such as Tsirelson's space $T$ [Ts] ; and finally, minimal spaces, such as $c_{0}$ or $\ell_{p}$, but also $T^{*}$, Schlumprecht's space $S$ [Sch1], or its dual $S^{*}[\mathrm{MP}$.

In [FR1] several other dichotomies for Banach spaces were obtained. The first one, called the third dichotomy, refines the distinction between the minimality of Rosenthal and strict quasi-minimality. Given a Banach space $X$ with a basis $\left(e_{n}\right)$, a space $Y$ is tight in $X$ if there is a sequence of successive subsets $I_{0}<I_{1}<I_{2}<\ldots$ of $\mathbb{N}$, such that the support on $\left(e_{n}\right)$ of any isomorphic copy of $Y$ intersects all but finitely many of the $I_{j}$ 's. In other words, for any infinite subset $J$ of $\mathbb{N}$,

$$
Y \nsubseteq\left[e_{i}: i \in \mathbb{N} \backslash \bigcup_{j \in J} I_{j}\right]
$$

where $\sqsubseteq$ means "embeds into".

The space $X$ itself is tight if all subspaces $Y$ of $X$ are tight in $X$.

As observed in [FG], the tightness of a space $Y$ in $X$ allows the following characterization: $Y$ is tight in $X$ if and only if

$$
\left\{u \in 2^{\omega}: Y \sqsubseteq\left[e_{n}: n \in u\right]\right\}
$$

is a meager subset of the Cantor space $2^{\omega}$. Here we identify the set ot subsets of $\omega$ with the Cantor space $2^{\omega}$, equipped with its usual topology.

After observing that the tightness property is hereditary and incompatible with minimality, the authors of [FR1] prove:

Theorem 1.3 (Third dichotomy [FR1]). Every Banach space contains a minimal subspace or a tight subspace.

Special types of tightness may be defined according to the way the $I_{n}$ 's may be chosen in function of $Y$. It is observed in [FR1] that the actual known examples of tight spaces satisfy one of two stronger forms of tightness, called by range, and with constants. Thus e.g. Gowers unconditional space $G_{u}$ is tight by range, and Tsirelson's space $T$ is tight with constants, see also [FR2] for other examples. 
We shall be mainly interested in tightness by range, which we define in the next subsection. We refer to the end of the paper for definitions and comments about tightness with constants.

\subsection{Ranges and supports.}

The following distinction is essential. If $X$ is a space with a basis $\left(e_{i}\right)_{i}$, then the definition of the support supp $x$ of a vector $x$ is well-known: it is the set $\{i \in \mathbb{N}$ : $\left.x_{i} \neq 0\right\}$, where $x=\sum_{i=0}^{\infty} x_{i} e_{i}$. On the other hand the range, ran $x$, of $x$ is the smallest interval of integers containing its support. So of course, having finite range and having finite support are the same, but the range is always an interval of integers, while the support may be an arbitrary subset of $\mathbb{N}$.

If $Y=\left[y_{n}, n \in \mathbb{N}\right]$ is a block subspace of $X$, then the support of $Y$ is $\cup_{n \in \mathbb{N}} \operatorname{supp} y_{n}$, and the range of $Y$ is $\cup_{n \in \mathbb{N}}$ ran $y_{n}$.

Let us now recall the criterion of Casazza, which appears in G1]. Two basic sequences $\left(x_{n}\right)_{n \in \mathbb{N}}$ and $\left(y_{n}\right)_{n \in \mathbb{N}}$ are said to be equivalent if the map $x_{n} \mapsto y_{n}$ extends to an isomorphism of $\left[x_{n}, n \in \mathbb{N}\right]$ onto $\left[y_{n}, n \in \mathbb{N}\right]$.

Proposition 1.4. [C] Let $X$ be a Banach space with a basis. Assume that for any block sequence $\left(x_{n}\right)$ in $X,\left(x_{2 n}\right)$ is not equivalent to $\left(x_{2 n+1}\right)$. Then $X$ is isomorphic to no proper subspace.

The criterion of Casazza leads to studying the possible isomorphisms between disjointly supported or disjointly ranged subspaces. As proved in [FR1], this turns out to have an essential connection with the notion of tightness. In what follows we shall say that two spaces are comparable if one embeds into the other.

If no two disjointly supported block-subspaces are isomorphic, then equivalently no two such subspaces are comparable. This is also equivalent to saying that for every block subspace $Y$, spanned by a block sequence $\left(y_{n}\right)$, the sequence of successive subsets $I_{0}<I_{1}<\ldots$ of $\mathbb{N}$ witnessing the tightness of $Y$ in $\left(e_{n}\right)$ may be defined by $I_{k}=\operatorname{supp} y_{k}$ for each $k$. When this happens it is said that $X$ is tight by support [FR1]. So Gowers' second dichotomy may be interpreted as a dichotomy between a form of tightness and a form of minimality, and $G_{u}$ is tight by support.

If now for every block subspace $Y=\left[y_{n}\right]$, the sequence of successive subsets $I_{0}<$ $I_{1}<\ldots$ of $\mathbb{N}$ witnessing the tightness of $Y$ in $\left(e_{n}\right)$ may be defined by $I_{k}=$ ran $y_{k}$ for each $k$, then $X$ is said to be tight by range. This is equivalent to no two block subspaces with disjoint ranges being comparable, a property which is formally weaker than tightness by support. Note that the criterion of Casazza applies to prove that a space which is tight by range cannot be isomorphic to its proper subspaces.

The distinction between range and support is relevant here. While it is easy to check that a basis which is tight by support must be unconditional, it is proved in FR2 that HI spaces may be tight by range; this is the case of an asymptotically unconditional and HI Gowers-Maurey's space $G$, due to Gowers [G2].

In [FR1] it was proved that there also exists a dichotomy relative to tightness by range. The authors define a space $X$ with a basis $\left(x_{n}\right)$ to be subsequentially minimal if 
every subspace of $X$ contains an isomorphic copy of a subsequence of $\left(x_{n}\right)$. Tsirelson's space $T$ is the classical example of subsequentially minimal, non-minimal space.

Theorem 1.5 (Fourth dichotomy [FR1]). Any Banach space contains a subspace with a basis which is either tight by range or subsequentially minimal.

The second case in Theorem 1.5 may be improved to the following hereditary property of a basis $\left(x_{n}\right)$, that is called sequential minimality: $\left(x_{n}\right)$ is quasi minimal and every block sequence of $\left[x_{n}\right]$ has a subsequentially minimal block sequence.

1.3. The list of $\mathbf{6}$ inevitable classes. The first four dichotomies and the interdependence of the properties involved can be visualized in the following diagram.

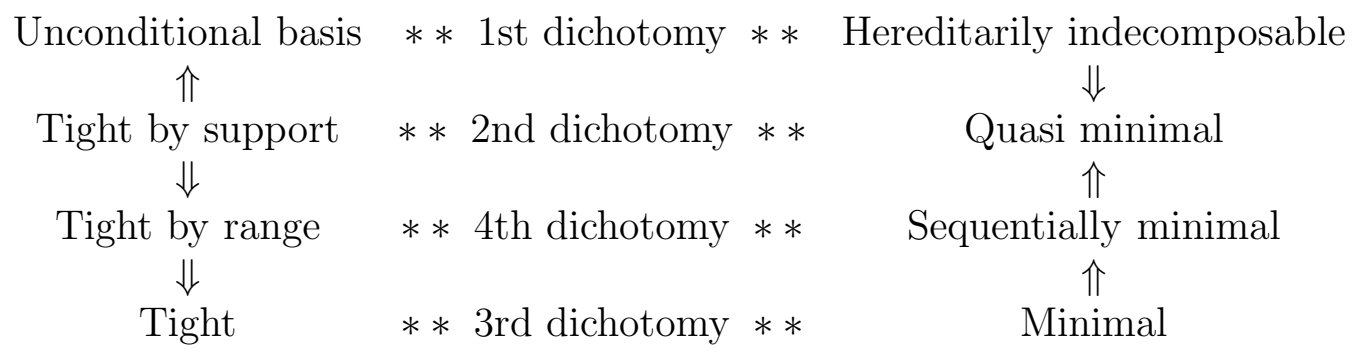

The easy observation that HI spaces are quasi-minimal is due to Gowers (see subsection 1.5). On the other hand it was shown in [GM, Corollary 19] and [GM, Theorem 21] that an HI space cannot be isomorphic to any proper subspace. This implies that an HI space cannot contain a minimal subspace.

Therefore by the third dichotomy, every HI space must contain a tight subspace, but it is unknown whether every HI space with a basis must itself be tight.

Combining the four dichotomies and the relations between them, the following list of 6 classes of Banach spaces contained in any Banach space is obtained in [FR1]:

Theorem 1.6. [FR1] Any infinite dimensional Banach space contains a subspace of one of the types listed in the following chart:

\begin{tabular}{|l|l|l|}
\hline Type & Properties & Examples \\
\hline (1) & HI, tight by range & $G, G^{*}$ \\
\hline (2) & HI, tight, sequentially minimal & $\mathcal{X}_{G M}$ \\
\hline (3) & tight by support & $G_{u}, G_{u}^{*}, X_{u}, X_{u}^{*}, X_{a b r}$ \\
\hline (4) & $\begin{array}{l}\text { unconditional basis, tight by range, } \\
\text { quasi minimal }\end{array}$ & ? \\
\hline (5) & $\begin{array}{l}\text { unconditional basis, tight, } \\
\text { sequentially minimal }\end{array}$ & $T, T^{(p)}$ \\
\hline (6) & unconditional basis, minimal & $S, S^{*}, T^{*}, c_{0}, \ell_{p}$ \\
\hline
\end{tabular}

For information about the examples appearing in type (1) and (3)-(6) we refer to [FR2]. Two major open problems of [FR1] were whether spaces of type (2) or 
(4) existed. The only known proofs of sequential minimality used properties which implied unconditionality, so presumably the construction of a type (2) space would require new methods.

The main result of this paper is the existence of an example $\mathcal{X}_{G M}$ of type (2), similar to Gowers-Maurey's space, which is reported on the chart above.

\subsection{The main result.}

Theorem 1.7. There exists a version $\mathcal{G M}$ of Gowers-Maurey's space such that

(a) $\mathcal{G} \mathcal{M}$ does not contain an unconditional basic sequence.

(b) Any block subspace of $\mathcal{G M}$ contains a block sequence $\left(y_{n}\right)_{n}$ such that $\left(y_{2 n}\right)$ is equivalent to $\left(y_{2 n+1}\right)$.

The proof of Theorem will be accomplished in Section 6, Theorem 6.2, The modification leading to $\mathcal{G M}$ is essentially technical. Note that by (a) and the first dichotomy, $\mathcal{G M}$ contains an HI subspace. So this subspace is not isomorphic to its proper subspaces, although by Theorem 1.7 (b), it does not satisfy Casazza's criterion. Using also the third and fourth dichotomy, we deduce that some subspace of $\mathcal{G M}$ satisfies:

Theorem 1.8. There exists a tight, HI, sequentially minimal space $\mathcal{X}_{G M}$.

It may be surprising to see that the answer to the existence of type (2) spaces is given by a non-essential modification of the first known example of HI space. We actually believe that $G M$ itself satisfies Theorem 1.7(b), and therefore fails to satisfy the criterion of Casazza.

We shall also observe that the space $\mathcal{G M}$ is locally minimal, which means that all finite dimensional subspaces of $\mathcal{G} \mathcal{M}$ embed into all its infinite dimensional subspaces, with uniform constant. Problem 5.2 from [FR2] asked whether a sequentially and locally minimal should be minimal or at least contain a minimal subspace. We therefore answer this by the negative.

Theorem 1.9. There exists a locally minimal, sequentially minimal, tight space.

To conclude this subsection let us mention that our results hold both in the real and in the complex setting.

\subsection{Some comments on our construction.}

Let us first recall why HI spaces are quasi-minimal. Let $X$ be an HI space with a basis. If $\epsilon>0$, and two block-subspaces $U$ and $V$ of $X$ are given, one can use the HI property to obtain two normalized block-sequences $\left(u_{n}\right)_{n}$ and $\left(v_{n}\right)_{n}$ in $U$ and $V$ respectively, so that $\left\|u_{n}-v_{n}\right\| \leq \epsilon 2^{-n}$ for all $n \in \mathbb{N}$. So there is a compact perturbation of the canonical injection mapping $\left[u_{n}, n \in \mathbb{N}\right]$ onto $\left[v_{n}, n \in \mathbb{N}\right]$, which are therefore isomorphic. Note that if $U$ and $V$ are disjointly supported, or even disjointly ranged, then each $u_{n}$ is disjointly supported from $v_{n}$. 
If now we want to obtain a canonical isomorphism between $\left[u_{n}, n \in \mathbb{N}\right]$ and $\left[v_{n}, n \in\right.$ $\mathbb{N}$ ], so that $\left(u_{n}\right)$ and $\left(v_{n}\right)$ are disjointly ranged and seminormalized block sequences, then such a crude approach does not work. Let us explain this when $\left(u_{n}\right)$ and $\left(v_{n}\right)$ are intertwined, i.e. $u_{0}<v_{0}<u_{1}<v_{1}<u_{2}<\cdots$. By using the projection on the range of $u_{n}$, we see that the norm $\left\|u_{n}-v_{n}\right\|$ is bounded below by a constant depending on the constant of the basis, and so the map $u_{n} \mapsto u_{n}-v_{n}$ can never be compact. We may however hope to pick $u_{n}$ and $v_{n}$ so that this map is strictly singular. Actually in the case when $X$ is, say, complex HI, we must do so. Indeed, we know in this case [F2 that there must exist $\lambda \in \mathbb{C}$ and a strictly singular operator $S:\left[u_{j}: j \in \mathbb{N}\right] \rightarrow X$, such that $v_{n}-\lambda u_{n}=S\left(u_{n}\right)$; so by projecting on ran $v_{n}$ we get that $S\left(u_{n}\right)$ is bounded below, and that $S$ is strictly singular non compact from $\left[u_{n}, n \in \mathbb{N}\right]$ into $X$.

So our result of existence of two intertwined and equivalent block sequences in any subspace of $\mathcal{G M}$ will be related to the techniques of the construction of strictly singular non-compact operators on subspaces of Schlumprecht's space $S$ and of $G M$ type spaces, as appears in $\mathrm{AS}$ ] and Sch2]. We shall replace the condition that $\left\|u_{n}-v_{n}\right\| \leq \epsilon 2^{-n}$ by the requirement that the sequence $u_{n}-v_{n}$ generates a spreading model which is "largely" dominated by the spreading models of $u_{n}, v_{n}$ and $u_{n}+v_{n}$. From some techniques of [Sch2], this will imply that the map taking $u_{n}$ to $u_{n}-v_{n}$ extends to a bounded (actually strictly singular) map, and the same for the map taking $v_{n}$ to $u_{n}-v_{n}$. Therefore $\left(u_{n}\right)$ and $\left(v_{n}\right)$ will be equivalent.

Note that our estimates will imply that $\left\|\sum_{i=1}^{k}\left(u_{n_{i}}-v_{n_{i}}\right)\right\| \leq \epsilon\left\|\sum_{i=1}^{k}\left(u_{n_{i}}+v_{n_{i}}\right)\right\|$ whenever $k<n_{1}<\cdots<n_{k}$ and $k$ is large enough with respect to $\epsilon$. Thus we recover the result of saturation of $G M$ with finite block-sequence $\left(y_{i}\right)_{i=1}^{2 k}$ such that $\left\|\sum_{i=1}^{2 k}(-1)^{i} y_{i}\right\| \leq \epsilon\left\|\sum_{i=1}^{2 k} y_{i}\right\|$, for some $k=k(\epsilon)$ large enough, but of course our result is much stronger, since we can choose $\left(y_{i}\right)$ to be any finite subsequence $\left(u_{n_{1}}, v_{n_{1}}, \ldots, u_{n_{k}}, v_{n_{k}}\right)$ as above. This estimate implies that $\mathcal{G} \mathcal{M}$ does not contain a subspace with an asymptotically unconditional basis, which means by Gowers' dichotomy that $\mathcal{G M}$ has a subspace which is HI. (and even, by [W], satisfies the HI property in a "uniform" way).

\subsection{Some preliminary definitions.}

We use the usual definitions and notation for $c_{00},\left(e_{i}\right), E(x), \operatorname{supp}(x), \operatorname{ran}(x)$, $E<F$ and $x<y$ for $E, F \subset \mathbb{N}$, and $x, y \in c_{00}$. The closed linear span of a basic sequence $\left(x_{n}\right)_{n \in \mathbb{N}}$ is denoted $\left[x_{n}, n \in \mathbb{N}\right]$.

We say that two vectors $x$ and $y$ in $c_{00}$ have the same distribution and write $x={ }_{\text {dist }} y$ if there there are natural numbers $l, m_{1}<m_{2}<\ldots m_{l}$, and $n_{1}<n_{2}<\ldots n_{l}$, and a sequence $\left(a_{i}: i=1,2 \ldots l\right) \subset \mathbb{R}$, so that

$$
x=\sum_{i=1}^{l} a_{i} e_{m_{i}} \text { and } y=\sum_{i=1}^{l} a_{i} e_{n_{i}} .
$$

We say $x$ is the distribution of $y$ if $x$ and $y$ have the same distribution and if the support of $x$ is an initial interval of $\mathbb{N}$.

Note that a vector $x \in c_{00}$ is uniquely defined by its distribution and its support. 
Definition 1.10. Let $X$ be a Banach space with a basis $\left(e_{i}\right)$. We call a vector $x$ in $X$ an $\ell_{1}^{+n}$-average, if $x=\frac{1}{n} \sum_{i=1}^{n} x_{i}$, where $\left(x_{i}\right)_{i=1}^{n}$ is a block sequence (of $\left(e_{i}\right)$ ) in $B_{X}$. For $c \in(0,1]$ an $\ell_{1}^{+n}$-average $x$ is called $\ell_{1}^{+n}$-average of constant $c$, if $\|x\| \geq c$.

If moreover $\left(x_{i}\right)_{i=1}^{n}$ is $\frac{1}{c}$-isomorphic to the $\ell_{1}^{n}$ unit vector basis, we say that $x$ is an $\ell_{1}^{n}$-average of constant $c$. In particular it follows in that case that $\left\|\sum_{i=1}^{n} \pm x_{i}\right\| \geq c$.

Remark. For a certain minor technical reason, we are not assuming in Definition 1.10 that the sequence $\left(x_{i}\right)$ is normalized. But of course if $x$ is supposed to be an $\ell_{1}^{+n}$ - or an $\ell_{1}^{n}$-average, of a constant $c$ close to 1 , then the norm of most of the $x_{i}$ also has to be close to 1 .

\section{THE SPACE $S$}

We recall the space introduced in [Sch1]. We define

$$
f(x)=\log _{2}(x+1), \text { for } x \geq 1 .
$$

The space $S$ is the completion of $c_{00}$ under the norm $\|\cdot\|_{S}$ which satisfies the following implicit equation.

$$
\|x\|_{S}=\max \left(\|x\|_{\infty}, \max _{\substack{l \in \mathbb{N} \\ E_{1}<E_{2}<\ldots E_{l}}} \frac{1}{f(l)} \sum_{j=1}^{l}\left\|E_{i}(x)\right\|_{S}\right) \text { for } x \in c_{00} .
$$

As observed in [Sch1], there is a norm $\|\cdot\|_{S}$ on $c_{00}$, which satisfies Equation (2), the completion $S$ of $\left(c_{00},\|\cdot\|_{S}\right)$ is reflexive, and $\left(e_{i}: i \in \mathbb{N}\right)$ is a 1 -subsymmetric (i.e 1-spreading and 1-unconditional) basis of $S$.

For $l=2,3 \ldots$ and $x \in S$ we define

$$
\|x\|_{l}=\frac{1}{f(l)} \max _{E_{1}<E_{2}<\ldots E_{l}} \sum_{j=1}^{l}\left\|E_{i}(x)\right\|_{S} .
$$

Then $\|\cdot\|_{l}$ is an equivalent norm on $S$ and for $x \in S$,

$$
\begin{aligned}
& \frac{1}{f(l)}\|x\| \leq\|x\|_{l} \leq\|x\| \text { and } \\
& \|x\|=\max \left(\|x\|_{\infty}, \sup _{l \in \mathbb{N}}\|x\|_{l}\right) .
\end{aligned}
$$

2.1. Upper bounds of $\|\cdot\|_{S}$. We will need to show some upper estimates for $\|\cdot\|_{S}$ and for basic sequences which have spreading models equivalent to the unit basis in $S$.

Definition 2.1. For a bounded sequence $\left(\xi_{i}\right)$ in $\mathbb{R}$ we denote the decreasing rearrangement of $\left(|\xi|_{i} \mid\right)$ by $\left(\xi_{i}^{\#}\right)$. 
Assume that $g:[1, \infty) \rightarrow[1, \infty)$ is an increasing function with $g(1)=1$. We define the following two norms on $c_{00}$. For $x=\left(x_{i}\right) \in c_{00}$ we define

$$
\|x\|_{g}=\max _{n_{1}<n_{2}<\ldots n_{l}, l \in \mathbb{N}} \frac{1}{g(l)} \sum_{i=1}^{l}\left|x_{n_{i}}\right| \text { and }\|x\|_{g}=\sum_{i=1}^{\infty} \frac{1}{g(i)} x_{i}^{\#} .
$$

It is clear that $\|\cdot\|_{g} \leq\|\cdot\|_{g}$. The following Lemma describes a situation in which we can bound $\|\cdot\|_{g^{p}}, 1<p<q$ by a multiple of $\|\cdot\|_{g^{q}}$.

Lemma 2.2. For $0<p<q$ there is a constant $C(p, q)$ so that

$$
\|x\|_{f^{q}} \leq C(p, q)\|x\|_{f^{p}} \text {, for all } x \in c_{00} .
$$

Here $f:[0, \infty) \rightarrow[0, \infty)$ is defined as in (1) by $f(x)=\log _{2}(x+1)$, for $x \geq 1$.

Proof. We first observe that

$$
C(p, q)=\sum_{n=1}^{\infty} \frac{f^{p}(n)-f^{p}(n-1)}{f^{q}(n)}<\infty .
$$

Indeed, by the Mean Value Theorem, there is for every $n \in \mathbb{N}$ an $\eta_{n} \in(n, n+1)$, so that

$$
f^{p}(n)-f^{p}(n-1)=\left.\left(\log _{2}(e)\right)^{p} \frac{\partial \ln ^{p}(x)}{\partial x}\right|_{x=\eta_{n}}=\left(\log _{2}(e)\right)^{p} p \frac{\ln ^{p-1}\left(\eta_{n}\right)}{\eta_{n}},
$$

and thus

$$
\left(\log _{2}(e)\right)^{q-p} \sum_{n=2}^{\infty} \frac{f^{p}(n)-f^{p}(n-1)}{f^{q}(n)}=\sum_{n=2}^{\infty} \frac{1}{\eta_{n}} \frac{1}{\ln ^{1-p}\left(\eta_{n}\right) \ln ^{q}(n+1)}<\sum_{n=2}^{\infty} \frac{1}{n} \frac{1}{\ln ^{1+q-p}(n)},
$$

which is finite by the integral test.

Secondly we claim that for $L \in \mathbb{N}$

$$
M_{L}=\max \left\{\|x\|_{f^{q}}: \operatorname{ran}(x) \subset[1, L], \text { and }\|x\|_{f^{p}} \leq 1\right\},
$$

is achieved for the vector

$$
x^{(L)}=\sum_{j=1}^{L}\left(f^{p}(j)-f^{p}(j-1)\right) e_{j} \text { with } f(0)=0,
$$

which would imply that $M_{L} \leq C(p, q)$. Indeed, $\left\|x^{(L)}\right\|_{f^{p}}=1$, and if $z=\left(z_{i}\right)_{i=1}^{L} \in \mathbb{R}^{L}$, $\|z\|_{f^{p}}=1$, and

$$
\|z\|_{f^{q}}=\sum_{j=1}^{L} \frac{z_{j}^{\#}}{f^{q}(j)}=M_{L},
$$

we can assume without loss of generality that $z_{1} \geq z_{2} \geq \ldots z_{L} \geq 0$. Note that actually $z_{L}>0$. Otherwise let $l_{0}=\min \left\{j: z_{i}=0\right.$ for all $\left.i \geq j\right\}$, and note for $l \geq l_{0}$ that

$$
\frac{1}{f^{p}(l)} \sum_{j=1}^{l} z_{j}=\frac{1}{f^{p}(l)} \sum_{j=1}^{l_{0}-1} z_{j}<\frac{1}{f^{p}\left(l_{0}-1\right)} \sum_{j=1}^{l_{0}} z_{j} \leq 1 .
$$

Thus we could increase the value of $z_{l_{0}}$, and thus increase the value of $\|z\|_{f^{q}}$, without increasing the value of $\|z\|_{f^{p}}$, which contradicts the maximality of $z$. 
We want to show now that $z=x^{(L)}$, which would imply our claim. If this were not true we put

$$
l_{o}=\min \left\{j \in\{1,2, \ldots, L\}: z_{j} \neq f^{p}(j)-f^{p}(j-1)\right\} .
$$

First we note that $z_{l_{0}}<f^{p}(j)-f^{p}(j-1)$, because otherwise $z_{l_{0}}>f^{p}(j)-f^{p}(j-1)$ and thus $\frac{1}{f^{p}\left(l_{0}\right)} \sum_{j=1}^{l_{0}} z_{j}>\frac{1}{f^{p}\left(l_{0}\right)} \sum_{j=1}^{l_{0}} f^{p}(j)-f^{p}(j-1)=1$,

Note that $l_{0} \neq L$ otherwise we could increase $z_{L}$ to $f^{p}(L)-f^{p}(L-1)$, which would not increase $\|z\|_{f^{p}}$, but certainly increase $\|z\|_{f^{q}}$. If $l_{0}<L$ we could increase $z_{l_{0}}$ by $\min \left(f^{p}\left(l_{0}\right)-f^{p}\left(l_{0}-1\right), z_{l_{0}}\right)>0$ and decrease $z_{l_{0}+1}$ by the same amount. This would

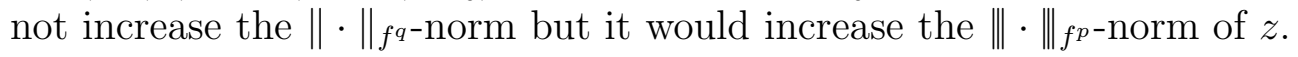

The proof of the next Lemma could be shown using [Sch2, Theorem 1.1] and its proof. Nevertheless, since the arguments in this case are much simpler we prefer to present a self contained argument.

Lemma 2.3. Let $\left(x_{n}\right)$ and $\left(y_{n}\right)$ be two basic seminormalized weakly null sequences in a Banach space $X$, having spreading models $E$ and $F$ with bases $\left(\tilde{x}_{n}\right)$ and $\left(\tilde{y}_{n}\right)$, respectively. Assume that for some $0<p<q$ and some $0<c, C<\infty$ it follows that

$$
\left\|\sum_{i=1}^{\infty} a_{n} \tilde{x}_{n}\right\|_{E} \geq c\left\|\sum_{i=1}^{\infty} a_{n} e_{n}\right\|_{f^{p}} \text { and }\left\|\sum_{i=1}^{\infty} a_{n} \tilde{y}_{n}\right\|_{F} \leq C\left\|\sum_{i=1}^{\infty} a_{n} e_{n}\right\|_{f^{q}} .
$$

Then there is a subsequence $\left(n_{k}\right)$ of $\mathbb{N}$ so that the map $x_{n_{k}} \mapsto y_{n_{k}}$ extends to a linear bounded operator.

Remark. Using the arguments in [Sch2] one can actually show that under the assumption of Lemma 2.3 there is a subsequence $\left(n_{k}\right)$ of $\mathbb{N}$ so that the map $x_{n_{k}} \mapsto y_{n_{k}}$ extends to a linear bounded and strictly singular operator.

Before proving Lemma 2.3 we will need the following

Lemma 2.4. Assume $0<p<q$ and define for $\varepsilon>0$

$$
\Delta_{(p, q)}(\varepsilon)=\sup \left\{\left\|\sum_{i=1}^{\infty} a_{i} e_{i}\right\|_{f^{q}}:\left|a_{i}\right| \leq \varepsilon, i=1,2 \ldots, \text { and }\left\|\sum_{i=1}^{\infty} a_{i} e_{i}\right\|_{f^{p}} \leq 1\right\} .
$$

Then

$$
\lim _{\varepsilon \searrow 0} \Delta_{(p, q)}(\varepsilon)=0 .
$$

Proof. Let $\eta>0$ be arbitrary and choose $n_{\eta} \in \mathbb{N}$ so that $\frac{1}{f^{q-p}(n)} \leq \eta$, for all $n \leq n_{\eta}$, and then choose

$$
\varepsilon=\eta \min _{n \leq n_{\eta}} \frac{f^{q}(n)}{n} .
$$

For any $\left(a_{i}\right) \in c_{00}$, with $\left|a_{i}\right| \leq \varepsilon$, for $i \in \mathbb{N}$, and $\left\|\sum_{i=1}^{\infty} a_{i} e_{i}\right\|_{f^{p}} \leq 1$ it follows therefore that, for some choice of $n \in \mathbb{N}$ and $i_{1}<i_{2}<\ldots i_{n}$ in $\mathbb{N}$, we have

$$
\left\|\sum_{i=1}^{\infty} a_{i} e_{i}\right\|_{f^{q}}=\frac{1}{f^{q}(n)} \sum_{s=1}^{n}\left|a_{i_{s}}\right| \leq \begin{cases}\frac{n}{f^{q}(n)} \leq \eta & \text { if } n \leq n_{\eta} \\ \frac{1}{f^{q-p}(n)}\left\|\sum_{i=1}^{\infty} a_{i} e_{i}\right\|_{f^{p}} \leq \eta & \text { if } n>n_{\eta},\end{cases}
$$

which verifies our claim. 
Proof of Lemma 2.3. We can assume that $\left(\tilde{y}_{n}\right)$ is not equivalent to the $c_{0}$ unit vector basis. Otherwise we may replace the norm on $\left[y_{n}: n \in \mathbb{N}\right]$ by

$$
\left\|\mid \sum a_{i} y_{i}\right\|=\left\|\sum a_{i} y_{i}\right\|+\left\|\sum a_{i} e_{i}\right\|_{f^{q}} \text { if }\left(a_{i}\right) \in c_{00} .
$$

We can therefore assume that for every $\varepsilon>0$ the number

$$
l(\varepsilon)=\max \left\{l: \begin{array}{l}
\text { there are }\left(a_{i}\right)_{i=1}^{l},\left|a_{i}\right| \geq \varepsilon, i=1,2 \ldots l, \text { and } \\
n_{1}<n_{2}<\ldots n_{l}, \text { so that }\left\|\sum_{i=1}^{l} a_{i} y_{n_{i}}\right\| \leq 1
\end{array}\right\}
$$

exists.

Let $r=(p+q) / 2$. By Lemma 2.4 we can choose a sequence $\left(\varepsilon_{n}\right) \subset(0,1)$ so that

$$
\sum_{n \in \mathbb{N}} \Delta_{(p, r)}\left(c \varepsilon_{n} / 6\right) \leq 1 \text { and } \sum_{n=1}^{\infty} n \varepsilon_{n} \leq 1 .
$$

Using the Schreier unconditionality of basic sequences Od] (see also [DOSZ for a more general statement), the fact that $\left(\tilde{x}_{n}\right)$ is the spreading model of $\left(x_{n}\right)$, and our assumption (5), we can assume, after passing to simultaneous subsequences of $\left(x_{n}\right)$ and $\left(y_{n}\right)$, if necessary, that for all $\left(a_{i}\right) \in c_{00}$ and all finite $F \subset \mathbb{N}$, with $n \leq \min F$ and $\# F \leq l\left(\varepsilon_{n+1}\right)$ we have

$$
\left\|\sum_{i \in F} a_{i} e_{i}\right\|_{f^{p}} \leq \frac{1}{c}\left\|\sum_{i \in F} a_{i} \tilde{x}_{i}\right\| \leq \frac{2}{c}\left\|\sum_{i \in F} a_{i} x_{i}\right\| \leq \frac{6}{c}\left\|\sum_{i=1}^{\infty} a_{i} x_{i}\right\|,
$$

and by using the fact that $\left(\tilde{y}_{n}\right)$ is the spreading model of $\left(y_{n}\right)$, our assumption (5), and Lemma 2.2, we can assume that for some constant $C_{3}$ and for all finite $F \subset \mathbb{N}$, with $n \leq \min F$ and $\# F \leq l\left(\varepsilon_{n+1}\right)$, and all $\left(a_{i}\right)_{i \in F}$ we have

$$
\left\|\sum_{i \in F} a_{i} y_{i}\right\| \leq 2\left\|\sum_{i \in F} a_{i} \tilde{y}_{i}\right\| \leq 2 C\left\|\mid \sum_{i \in F} a_{i}\right\|\left\|_{f^{q}} \leq C_{3}\right\| \sum_{i \in F} a_{i} e_{i} \|_{f^{r}} .
$$

By Elton's near unconditionality [El] (see also [DOSZ, Theorem 6]) and the fact that $l\left(\varepsilon_{1}\right)$ is finite we can assume, after passing to subsequences, if necessary, that there are constants $C_{1}$ and $C_{2}$ so that for every $\left(a_{i}\right) \in c_{00}$, with $\left\|\sum a_{i} x_{i}\right\| \leq 1$, it follows that

$$
\left\|\sum_{j=1,\left|a_{j}\right|>\varepsilon_{1}}^{\infty} a_{j} y_{j}\right\| \leq C_{1}\left\|\sum_{j=1,\left|a_{j}\right|>\varepsilon_{1}}^{\infty} a_{j} x_{j}\right\| \leq C_{2}\left\|\sum_{j=1}^{\infty} a_{j} x_{j}\right\| \leq C_{2} .
$$

Now let $\left(a_{j}\right) \in c_{00}$ and assume that $\left\|\sum_{i=1}^{n} a_{i} x_{i}\right\|=1$. Then, by (11),

$$
\left\|\sum_{j=1}^{\infty} a_{j} y_{j}\right\| \leq\left\|\sum_{\left|a_{j}\right| \leq \varepsilon_{1}} a_{j} y_{j}\right\|+\left\|\sum_{\left|a_{j}\right|>\varepsilon_{1}} a_{j} y_{j}\right\| \leq\left\|\sum_{\left|a_{j}\right| \leq \varepsilon_{1}}^{\infty} a_{j} y_{j}\right\|+C_{2}\left\|\sum_{j=1}^{\infty} a_{j} x_{j}\right\|
$$


and

$$
\begin{aligned}
\left\|\sum_{j=1,\left|a_{j}\right| \leq \varepsilon_{1}}^{\infty} a_{j} y_{j}\right\| & \leq \sum_{n=1}^{\infty}\left\|\sum_{\varepsilon_{n+1}<\left|a_{j}\right| \leq \varepsilon_{n}} a_{j} y_{j}\right\| \\
& \leq \sum_{n=1}^{\infty}\left[n \varepsilon_{n}+\left\|\sum_{n<j, \varepsilon_{n+1}<\left|a_{j}\right| \leq \varepsilon_{n}} a_{j} y_{j}\right\|\right] \\
& \left.\leq 1+C_{3} \sum_{n=1}^{\infty}\left\|\sum_{n<j, \varepsilon_{n+1}<\left|a_{j}\right| \leq \varepsilon_{n}} a_{j} e_{j}\right\|_{f^{r}} \text { (by (8) and (10) ) }\right) \\
& =1+\frac{6 C_{3}}{c} \sum_{n=1}^{\infty}\left\|\sum_{n<j, \varepsilon_{n+1}<\left|a_{j}\right| \leq \varepsilon_{n}} \frac{c}{6} a_{j} e_{j}\right\|_{f^{r}} .
\end{aligned}
$$

Now it follows from (9) that

$$
\left\|\sum_{n<j, \varepsilon_{n+1}<\left|a_{j}\right| \leq \varepsilon_{n}} \frac{c}{6} a_{j} e_{j}\right\|_{f^{p}} \leq 1
$$

and thus (8) and the definition of $\Delta_{p, r}$ yield that

$$
\left\|\sum_{j=1,\left|a_{j}\right| \leq \varepsilon_{1}}^{\infty} a_{j} y_{j}\right\| \leq 1+\frac{6 C_{3}}{c} .
$$

We proved therefore that if $\left\|\sum_{i=1}^{\infty} a_{i} x_{i}\right\| \leq 1$ then

$$
\left\|\sum_{i=1}^{\infty} a_{i} y_{i}\right\| \leq C_{2}+1+\frac{6 C_{3}}{c}
$$

which finishes the proof of our claim.

We finally want to compare the norms $\|\cdot\| \|_{f}$ and $\|\cdot\|_{S}$ and first prove the following Lemma.

Lemma 2.5. For every $x^{*}=\left(\xi_{j}\right) \in B_{S^{*}}$ and $n \in \mathbb{N}$, we have that

$$
\xi_{n}^{\#} \leq \frac{1}{f(n)} .
$$

Proof. By the 1-unconditionality of both norms in $S$ and $S^{*}$ we need to prove (12) only for non negative sequences $x^{*}=\left(\xi_{j}\right)$ in $c_{00}$. Let $E=\left\{j_{1}, j_{2}, \ldots, j_{n}\right\} \subset \mathbb{N}$ have $n$ elements, so that

$$
\xi_{j_{1}} \geq \xi_{j_{2}} \geq \ldots \xi_{j_{n}}=\xi_{n}^{\#}
$$

and put

$$
y^{*}=\xi_{n}^{\#} \sum_{s=1}^{n} e_{j_{s}}^{*}
$$


Since $S^{*}$ is supression 1 -unconditional, it follows that $\left\|y^{*}\right\| \leq 1$, and since

$$
y=\frac{f(n)}{n} \sum_{s=1}^{n} e_{j_{s}} \in S_{S}
$$

(see [Sch1]) it follows that

$$
1 \geq\left\langle y^{*}, y\right\rangle=f(n) \xi_{n}^{\#}
$$

which proves our claim.

Corollary 2.6. For $x \in c_{00}$ we have

$$
\|x\|_{S} \leq\|x\|_{f}
$$

\subsection{Yardstick vectors.}

The following type of vectors were introduced in $[\mathrm{KL}]$.

Definition 2.7. (Yardstick Vectors)

We call a finite or infinite sequence of natural number $m_{1}, m_{2}, m_{3}, \ldots$ admissi$b l e$, if for any $i$, for which $m_{i}$ exists, $m_{i}$ is even and is a multiple of the product $\prod_{A \subset 1,2, \ldots i-1}\left(\sum_{j \in A} m_{j}\right)$ (as usual $\prod_{\emptyset}=1$ ). Note that any subsequence of admissible sequences is also admissible.

By induction we define the vector $y\left(m_{1}, m_{2}, \ldots m_{k}\right)$ for each $k$ and each admissible finite sequence $\left(m_{1}, m_{2}, \ldots, m_{k}\right) \subset \mathbb{N}$; the support of $y\left(m_{1}, m_{2}, \ldots m_{k}\right)$ will be the interval $\left[1, \sum_{i=1}^{k} m_{i}\right]$.

If $k=1$ we put for $m \in \mathbb{N}$

$$
y(m)=\frac{f(m)}{m} \sum_{i=1}^{m} e_{i} .
$$

Assume that $y\left(m_{1}, m_{2}, \ldots m_{k^{\prime}}\right)$ has been defined for each $k^{\prime}<k$ and each admissible sequence $\left(m_{1}, m_{2}, \ldots, m_{k^{\prime}}\right) \subset \mathbb{N}$.

From our induction hypothesis the support of $y\left(m_{1}, m_{2}, \ldots, m_{k-1}\right)$ is $\left[1, m_{1}+m_{2}+\right.$ $\left.\ldots+m_{k-1}\right]$ and we write $y\left(m_{1}, m_{2}, \ldots, m_{k-1}\right)$ as

$$
y\left(m_{1}, m_{2}, \ldots, m_{k-1}\right)=\sum_{i=1}^{m_{1}+m_{2}+\ldots+m_{k-1}} a_{i} e_{i} .
$$

Now we define $\tilde{y}$ to be the vector, which has the same distribution as $y\left(m_{1}, m_{2}, \ldots, m_{k-1}\right)$, and whose support is

$$
\operatorname{supp}(\tilde{y})=\left\{1+(i-1) \frac{m_{k}}{m_{1}+m_{2}+\ldots m_{k-1}}: i=1,2 \ldots, \sum_{j=1}^{k-1} m_{j}\right\}
$$

(i.e we spread out the coordinates of $y\left(m_{1}, m_{2}, m_{k-1}\right)$, so that between any two successive non zero coordinates there are $\frac{m_{k}}{m_{1}+m_{2}+\ldots m_{k-1}}$ zeros).

Then we define

$$
y\left(m_{1}, m_{2}, \ldots, m_{k}\right)=\tilde{y}+\frac{f\left(m_{k}\right)}{m_{k}} \sum_{i \in\left[1, m_{1}+m_{2}++\ldots m_{k}\right] \backslash \operatorname{supp}(\tilde{y})} e_{i}
$$


(i.e. we are replacing the zeros on the interval $\left[1, m_{1}+m_{2}+\ldots m_{k}\right]$ by the value $\left.\left.f\left(m_{k}\right) / m_{k}\right)\right)$. So, for example, $y\left(m_{1}\right)$ and $y\left(m_{1}, m_{2}\right)$ are the following vectors:

$$
\begin{aligned}
y\left(m_{1}\right) & =\underbrace{\left(\frac{f\left(m_{1}\right)}{m_{1}}, \frac{f\left(m_{1}\right)}{m_{1}}, \ldots, \frac{f\left(m_{1}\right)}{m_{1}}\right)}_{m_{1} \text { times }} \\
y\left(m_{1}, m_{2}\right) & =\underbrace{(\frac{f\left(m_{1}\right)}{m_{1}}, \underbrace{\frac{f\left(m_{2}\right)}{m_{2}}, \ldots, \frac{f\left(m_{2}\right)}{m_{2}}}_{m_{2} / m_{1} \text { times }}, \ldots, \frac{f\left(m_{1}\right)}{m_{1}}, \underbrace{\frac{f\left(m_{2}\right)}{m_{2}}, \ldots, \frac{f\left(m_{2}\right)}{m_{2}}}_{m_{2} / m_{1} \text { times }}) .}_{m_{1} \text { times }}
\end{aligned}
$$

If $\bar{x}=\left(x_{n}\right)_{n \in \mathbb{N}}$ is a block sequence in $c_{00}$, and if $\left(m_{1}, \ldots m_{k}\right) \subset \mathbb{N}$ is admissible, we define $y_{\bar{x}}\left(m_{1}, m_{2}, \ldots, m_{k}\right)$ to be a linear combination of the $x_{n}$ ' $\mathrm{s}$ with the same distribution as $y\left(m_{1}, m_{2}, \ldots, m_{k}\right)$ has on the $e_{n}$ 's, i.e.

$$
y_{\bar{x}}\left(m_{1}, m_{2}, \ldots, m_{k}\right)=\sum_{i \in \operatorname{supp}\left(y\left(m_{1}, m_{2}, \ldots, m_{k}\right)\right)} a_{i} x_{i},
$$

where the $a_{i}$ are such that

$$
y\left(m_{1}, m_{2}, \ldots, m_{k}\right)=\sum_{i \in \operatorname{supp}\left(y\left(m_{1}, m_{2}, \ldots, m_{k}\right)\right)} a_{i} e_{i} .
$$

It follows from the arguments in $\mathrm{KL}$ that for $k \in \mathbb{N}$ and $\varepsilon>0$ one can find $m_{1}<m_{2}<\ldots m_{k}$ in $\mathbb{N}$ so that $\left\|y\left(m_{1}, m_{2}, \ldots m_{k}\right)\right\|_{S} \leq 1+\varepsilon$. Since $y\left(m_{1}, m_{2}, \ldots m_{k}\right)$ is the sum of disjointly supported vectors $z_{1}, z_{2}, \ldots z_{k}$, with $z_{i}$ having the same distribution as $\frac{f\left(m_{i}\right)}{m_{i}} \sum_{j=1}^{m_{i}} e_{j}$, for $i=1,2 \ldots k$, (and thus $\left\|z_{j}\right\|=1$, by [Sch1]), it follows that $\ell_{\infty}^{k}, k \in \mathbb{N}$ are uniformly represented in $S$. Something stronger is true. Using similar arguments as in [KL] it is actually possible to prove under appropriate growth conditions on $\left(m_{i}\right)$ that the sequence $\left(y\left(m_{1}, m_{2}, \ldots m_{k}\right): k \in \mathbb{N}\right)$ is uniformly bounded in $S$. For completeness we will present a self contained proof of this fact. First we prove the following lemma, which will serve as the induction step for choosing the sequence $\left(m_{i}\right)$.

Lemma 2.8. Assume we are given $k, m \in \mathbb{N}$, with $k<m, c \geq 1$ and some $\varepsilon \in$ $(0, f(2)-1) / f(2))$ satisfying the following conditions:

$m$ is divisible by $k$

$$
f(m) \geq C \max \left(\frac{50}{\varepsilon^{2}}, \frac{f\left(l_{0}\right) f\left(l_{0}-1\right)}{f\left(l_{0}\right)-f\left(l_{0}-1\right)}\right)
$$

where $l_{0}=\min \{l \in \mathbb{N}: f(l) \geq 6\}$

$$
\frac{f(m)}{f(m / k)} \leq 1+\frac{\varepsilon}{6}
$$


Assume further $\left(j_{s}\right)_{s=1}^{m} \subset \mathbb{N}$ and $\left(x_{s}\right)_{s=1}^{m} \subset S$ have the property that

$$
\begin{aligned}
& e_{j_{1}}<x_{1}<e_{j_{2}}<x_{2} \ldots e_{j_{m-1}}<x_{m-1}<e_{j_{m}}<x_{m}, \text { and } \\
& \left\|x_{s}\right\| \leq \frac{C}{m}, \text { for } s=1,2 \ldots m
\end{aligned}
$$

Then it follows that

$$
\begin{aligned}
& \left\|\sum_{s=1}^{m} \frac{f(m)}{m} e_{j_{s}}+x_{s}\right\|_{S} \leq C(1+\varepsilon) \text { and } \\
& \left\|\sum_{s=(i-1)(m / k)+1}^{i m / k} \frac{f(m)}{m} e_{j_{s}}+x_{s}\right\|_{S} \leq \frac{C(1+\varepsilon)}{k} \text { for } i=1,2,3, \ldots, k .
\end{aligned}
$$

In particular the vectors

$$
y_{i}=\frac{k}{C(1+\varepsilon)} \sum_{s=(i-1)(m / k)+1}^{i m / k} \frac{f(m)}{m} e_{j_{s}}+x_{s}, \text { for } i=1,2, \ldots, k
$$

are in $B_{S}$ and $\left(y_{i}\right)$ is $C(1+\varepsilon)$-equivalent to the unit vector basis in $\ell_{1}^{k}$.

Proof. We note that for any scalars $\left(a_{i}\right)_{i=1}^{k}$, we have

$$
\left\|\sum_{i=1}^{k} a_{i} y_{i}\right\| \geq f(m)^{-1}\left(\sum_{s=1}^{m} e_{j_{s}}^{*}\right)\left(\sum_{i=1}^{k} a_{i} y_{i}\right)=f(m)^{-1} \sum_{i=1}^{k} a_{i}\left(\sum_{s=(i-1)(m / k)+1}^{i m / k} e_{j_{s}}^{*}\left(y_{i}\right)\right) .
$$

It follows easily, assuming (20) and using the 1-unconditionality of the basis, that $\left(y_{i}\right)$ is $C(1+\varepsilon)$-equivalent to the unit vector basis in $\ell_{1}^{k}$.

To prove (19) and (20) we put

$$
x=\sum_{s=1}^{m} \frac{f(m)}{m} e_{j_{s}}+x_{s}
$$

We will proof by induction for each $n \in\{1,2, \ldots m\}$, that whenever $0 \leq s_{0}<s_{1} \leq m$, with $s_{1}-s_{0}=n$, and $I \subset \mathbb{N}$ is an interval with $j_{s_{0}}<I<j_{s_{1}+1}$ (where we let $j_{0}=0$ and $\left.j_{s_{m+1}}=\infty\right)$, then

$$
\|I(x)\| \leq \frac{f(m)}{m} \frac{n}{f(n)} C\left(1+\frac{\varepsilon}{3}\right)+\frac{n}{m} C \frac{\varepsilon}{3} .
$$


From that we deduce (19) by letting $I=\mathbb{N}$ and $n=m$. Moreover, if we put $I=$ $\left[j_{(i-1)(m / k)+1}, j_{i(m / k)+1}-1\right]$, for $i=1,2 \ldots k$, we deduce from (21) and (16) that

$$
\begin{aligned}
\left\|\sum_{s=(i-1)(m / k)+1}^{i m / k} \frac{f(m)}{m} e_{j_{s}}+x_{s}\right\| & =\|I(x)\| \\
& \leq \frac{f(m)}{m} \frac{m / k}{f(m / k)} C\left(1+\frac{\varepsilon}{3}\right)+\frac{m / k}{m} C \frac{\varepsilon}{3} \\
& =\frac{1}{k} \frac{f(m)}{f(m / k)} C\left(1+\frac{\varepsilon}{3}\right)+\frac{1}{k} C \frac{\varepsilon}{3} \leq \frac{1}{k} C(1+\varepsilon)
\end{aligned}
$$

which implies (20).

First let $n \in \mathbb{N}$, so that $f(n) \leq \frac{6}{\varepsilon}$, and let $I \subset \mathbb{N}$ be an interval with $j_{s_{0}}<I<j_{s_{1}+1}$ for some choice of $s_{0}, s_{1} \in\{1,2 \ldots, m\}$, and $s_{1}-s_{0}=n, l \geq 2$. Then

$$
\begin{aligned}
\|I(x)\|_{l} & \leq\left\|\sum_{s=s_{0}+1}^{s_{1}} \frac{f(m)}{m} e_{j_{s}}\right\|+\left\|\sum_{s=s_{0}}^{s_{1}} x_{s}\right\| \\
& \leq \frac{f(m)}{m} \frac{n}{f(n)}+C \frac{n+1}{m} \\
& =\frac{f(m)}{m} \frac{n}{f(n)}\left[1+C \frac{n+1}{n} \frac{f(n)}{f(m)}\right] \\
& \leq \frac{f(m)}{m} \frac{n}{f(n)} C\left[1+\frac{12}{\varepsilon f(m)}\right] \leq \frac{f(m)}{m} \frac{n}{f(n)} C\left[1+\frac{\varepsilon}{3}\right] \text { (by (15) ) }
\end{aligned}
$$

which implies our claim for $n \in \mathbb{N}$, for which $f(n) \leq \frac{6}{\varepsilon}$.

Assume that our induction hypothesis is true for all $n^{\prime}<n, n \in \mathbb{N}$, with $f(n)>6 / n$, and all intervals $I \subset \mathbb{N}$ for which there are $j_{s_{0}}<I<j_{s_{1}+1}$ with $0 \leq s_{0}<s_{1} \leq m$ and $s_{1}-s_{0}=n$.

Let $l \in \mathbb{N}, l \geq 2$, such that $\|x\|=\|x\|_{l}$ (since $n \geq 2$ it follows that $\|I(x)\|_{\infty}<$ $\|I(x)\|_{2}$, and thus $\left.l \geq 2\right)$.

We choose numbers $l_{1}$ and $l_{2}$ in $\mathbb{N} \cup\{0\}$, with $l=l_{1}+l_{2}$, and intervals $E_{1}^{(1)}<$ $E_{2}^{(1)}, \ldots E_{l_{1}}^{(1)}$ and $E_{1}^{(2)}<E_{2}^{(2)}, \ldots E_{l_{1}}^{(2)}$, so that

$$
\bigcup_{t=1}^{l_{1}} E_{t}^{(1)} \cap \bigcup_{t=1}^{l_{1}} E_{t}^{(2)}=\emptyset \text { and } \bigcup_{t=1}^{l_{1}} E_{t}^{(1)} \cup \bigcup_{t=1}^{l_{1}} E_{t}^{(2)}=I \text {, }
$$

and so that each of the $E_{t}^{(1)}$ contains at least one of the $j_{s}, s_{0}<s \leq s_{1}$ and none of the $E_{t}^{(2)}$ intersects with $\left\{j_{s_{0}+1}, j_{s_{0}+2}, \ldots j_{s_{1}}\right\}$, and so that

$$
\|I(x)\|_{l}=\frac{1}{f(l)}\left(\sum_{t=1}^{l_{1}}\left\|E_{t}^{(1)}(x)\right\|+\sum_{t=1}^{l_{2}}\left\|E_{t}^{(2)}(x)\right\|\right) .
$$


We note that $l_{1} \geq 2$, otherwise it would follow that $l_{1}=1$ and for all $t=1,2 \ldots l_{2}$ either $j_{s_{0}}<E_{t}^{(2)}<j_{s_{0}+1}$ or $j_{s_{1}}<E_{t}^{(2)}<j_{s_{1}+1}$, and thus, by (18)

$$
\|I(x)\|=\|I(x)\|_{l} \leq \frac{2 C}{m}+\frac{1}{f(l)}\left\|E_{1}^{(1)}(x)\right\| \leq \frac{2 C}{m}+\frac{1}{f(2)}\|I(x)\|,
$$

and thus

$$
\frac{f(m)}{m}\left(1-\frac{1}{f(l)}\right) \leq \frac{2 C}{m}
$$

which contradicts (15) and the restrictions on $\varepsilon$.

We can therefore apply our induction hypothesis and deduce that there are numbers $s_{0}=\tilde{s}_{0}<\tilde{s}_{1}<\ldots \tilde{s}_{l_{1}}=s_{1}$ so that for $t=1,2, \ldots l_{1}$

$$
\left\|E_{t}^{(1)}(x)\right\| \leq \frac{f(m)}{m} \frac{\tilde{s}_{t}-\tilde{s}_{t-1}}{f\left(\tilde{s}_{t}-\tilde{s}_{t-1}\right)} C\left(1+\frac{\varepsilon}{3}\right)+\frac{\tilde{s}_{t}-\tilde{s}_{t-1}}{m} C \frac{\varepsilon}{3} .
$$

Moreover it follows that

$$
\frac{1}{f(l)} \sum_{t=1}^{l_{2}}\left\|E_{t}^{(2)}(x)\right\| \leq\left\|\sum_{s=s_{0}}^{s_{1}} x_{s}\right\| \leq C \frac{n+1}{m} .
$$

Case 1 . If $6 \leq f(l)$, then we deduce that

$$
\begin{aligned}
\|I(x)\|_{l} & =\frac{1}{f(l)}\left(\sum_{t=1}^{l_{1}}\left\|E_{t}^{(1)}(x)\right\|+\sum_{t=1}^{l_{2}}\left\|E_{t}^{(2)}(x)\right\|\right) \\
& \leq C \frac{n+1}{m}+\frac{1}{f(l)} C \sum_{t=1}^{l_{1}}\left[\frac{f(m)}{m} \frac{\tilde{s}_{t}-\tilde{s}_{t-1}}{f\left(\tilde{s}_{t}-\tilde{s}_{t-1}\right)}\left(1+\frac{\varepsilon}{3}\right)+\frac{\tilde{s}_{t}-\tilde{s}_{t-1}}{m} \frac{\varepsilon}{3}\right] \\
& \leq C \frac{n+1}{m}+\frac{1}{f(l)} C\left[\frac{f(m)}{m} \frac{n}{f\left(n / l_{1}\right)}\left(1+\frac{\varepsilon}{3}\right)+\frac{n}{m} \frac{\varepsilon}{3}\right]
\end{aligned}
$$

(By the concavity of the map $\xi \mapsto \xi / f(\xi)$ )

$$
\begin{aligned}
& =\frac{f(m)}{m} C \frac{n}{f(l) f\left(n / l_{1}\right)}\left(1+\frac{\varepsilon}{3}\right)+C \frac{n}{m}\left[1+\frac{1}{n}+\frac{\varepsilon}{3 f(l)}\right] \\
& \leq \frac{f(m)}{m} C \frac{n}{f(n)}\left(1+\frac{\varepsilon}{3}\right)+C \frac{n}{m}\left(1+\frac{\varepsilon}{3}\right)
\end{aligned}
$$

where the last inequality follows from the fact that $f(a / b) f(b) \geq f(a)$ for $a, b \geq 2$ (see [Sch1]) and (15). This finishes the proof of our induction step in this case. 
Case 2. If $f(l)<6$ we claim that $l_{2}=0$. Indeed, otherwise $l=l_{1}+l_{2} \geq 3$ (we already observed that $l_{1} \geq 2$ ) and

$$
\begin{aligned}
\|I(x)\|_{l-1} & \geq \frac{1}{f(l-1)}\left[\sum_{t=1}^{l_{1}}\left\|E_{t}^{(1)}\right\|+\sum_{t=2}^{l_{2}}\left\|E_{t}^{(2)}(x)\right\|\right] \\
& \geq \frac{1}{f(l)}\left[\sum_{t=1}^{l_{1}}\left\|E_{t}^{(1)}\right\|+\sum_{t=2}^{l_{2}}\left\|E_{t}^{(2)}(x)\right\|\right]+\left(\frac{1}{f(l-1)}-\frac{1}{f(l)}\right) \frac{f(m)}{m} \\
& >\frac{1}{f(l)}\left[\sum_{t=1}^{l_{1}}\left\|E_{t}^{(1)}\right\|+\sum_{t=2}^{l_{2}}\left\|E_{t}^{(2)}(x)\right\|\right]+\frac{1}{f(l)}\left\|E_{1}^{(2)}(x)\right\|(\text { by (15) }) \\
& =\|I(x)\|_{l},
\end{aligned}
$$

which contradicts the assumption that $\|I(x)\|=\|I(x)\|_{l}$. So it follows that $l=l_{1}$ and from (23) and the concavity of the map $\xi \mapsto f(\xi) / \xi, \xi \geq 1$ it follows therefore that

$$
\begin{aligned}
\|I(x)\|_{l} & \leq \frac{1}{f\left(l_{1}\right)} \sum_{t=1}^{l_{1}}\left[\frac{f(m)}{m} \frac{\tilde{s}_{t}-\tilde{s}_{t-1}}{f\left(\tilde{s}_{t}-\tilde{s}_{t-1}\right)} C\left(1+\frac{\varepsilon}{3}\right)+\frac{\tilde{s}_{t}-\tilde{s}_{t-1}}{m} C \frac{\varepsilon}{3}\right] \\
& \leq \frac{f(m)}{m} \frac{n}{f(l) f(n / l)} C\left(1+\frac{\varepsilon}{3}\right)+\frac{n}{m} C \frac{\varepsilon}{3} \leq \frac{f(m)}{m} \frac{n}{f(n)} C\left(1+\frac{\varepsilon}{3}\right)+\frac{n}{m} C \frac{\varepsilon}{3},
\end{aligned}
$$

which finishes the proof of the inductions step the the proof of our lemma.

Lemma 2.9. Assume that $\left(\varepsilon_{i}\right) \subset(0,(f(2)-1) / f(2))$ is summable, and put $C_{i}=$ $\prod_{j \geq i}\left(1+\varepsilon_{j}\right)$, for $i \in \mathbb{N} \cap\{0\}$.

Assume that the sequence $\left(m_{i}: i \in \mathbb{N} \cup\{0\}\right) \subset \mathbb{N}$ is an admissible sequence and satisfies the following growth conditions. For all $i \in \mathbb{N}$ we assume that

$$
f\left(m_{i}\right) \geq C_{i} \max \left(\frac{50}{\varepsilon_{i}^{2}}, \frac{f\left(l_{0}\right) f\left(l_{0}-1\right)}{f\left(l_{0}\right)-f\left(l_{0}-1\right)}\right),
$$

where $l_{0}=\min \{l \in \mathbb{N}: f(l) \geq 6\}$, and

$$
\frac{f\left(m_{i}\right)}{f\left(m_{i} / m_{i-1}\right)} \leq 1+\frac{\varepsilon_{i}}{6}
$$

Then it follows for all $i \leq j$ in $\mathbb{N}$ that

$$
\begin{aligned}
& \left\|y\left(m_{i}, m_{i+1}, \ldots m_{j}\right)\right\| \leq C_{i} \text {, and } \\
& \frac{1}{C_{i}} y\left(m_{i}, m_{i+1}, \ldots m_{j}\right) \text { is an } \ell_{1}^{m_{i-1}} \text {-average of constant } 1 / C_{i} .
\end{aligned}
$$

Remark. For the sequence $\left(m_{i}\right)$ as chosen in Lemma 2.9 we deduce therefore that, if $k \in \mathbb{N}$ and $\varepsilon>0$ and if $i_{0} \in \mathbb{N}$ is chosen so that $k \leq m_{i_{0}}$ and $\prod_{i=i_{0}}^{\infty}\left(1+\varepsilon_{i}\right) \leq 1+\varepsilon$, 
then for all sequences $i_{0}<i_{1}<i_{1}<\cdots<i_{l}, l \in \mathbb{N}$, it follows that

$$
\begin{aligned}
& \left\|y\left(m_{i_{1}}, m_{i_{2}}, \ldots, m_{i_{l}}\right)\right\|_{S} \leq 1+\varepsilon \text { and } \\
& \frac{1}{1+\varepsilon} y\left(m_{i_{1}}, m_{i_{2}}, \ldots, m_{i_{l}}\right) \text { is an } \ell_{1}^{m_{i_{0}}} \text {-average of constant } \frac{1}{1+\varepsilon} .
\end{aligned}
$$

Proof of Lemma [2.9. Let the sequence $\left(m_{j}: j \in \mathbb{N} \cup\{0\}\right)$ be chosen as required. Let $j \in \mathbb{N}$. By induction on $i=0,1,2 \ldots, j-1$ we will show that

$$
\begin{aligned}
& \left\|y\left(m_{j-i}, m_{j-i+1}, \ldots, m_{j}\right)\right\| \leq C_{j-i} \text {, and } \\
& \frac{1}{C_{j-i}} y\left(m_{j-i}, m_{j-i+1}, \ldots m_{j}\right) \text { is an } \ell^{m_{j-i-1} \text {-average of constant } 1 / C_{j-i} .}
\end{aligned}
$$

More precisely, we can write $y=y\left(m_{j-i}, m_{2}, \ldots m_{j}\right)$ as

$$
y=\frac{C_{j-i}}{m_{j-i-1}} \sum_{s=1}^{m_{j-i-1}} y_{s} \text { where } y_{1}<y_{2}<\ldots y_{m_{j-i-1}} \text { are in } B_{S} \text {, }
$$

equally distributed and $C_{j-i}$-equivalent to the basis of $\ell_{1}^{m_{j-i-1}}$.

For $i=0$ it follows that $y\left(m_{j}\right)=\frac{f\left(m_{j}\right)}{m_{j}} \sum_{s=1}^{m_{j}} e_{s} \in S_{S}$, and the conditions of Lemma 2.8 are satisfied with $m=m_{j}, \varepsilon=\varepsilon_{j}$, and $C=1 \leq C_{j+1}, k=m_{j-1}$, and $x_{s}=0$, for $s=0,1, \ldots m_{j}$. Since $C_{j}=\left(1+\varepsilon_{j}\right) C_{j+1}$, this implies our claim for $i=0$.

Assuming (31) and (32) are true for $i-1$ with $1 \leq i<j-1$. Using the recursive definition of $y\left(m_{j-i}, m_{j-i+1}, \ldots m_{j}\right)$ one can write it as

$$
y\left(m_{j-i}, m_{j-i+1}, \ldots, m_{j}\right)=\sum_{s=1}^{m_{j-i}} \frac{f\left(m_{j-i}\right)}{m_{j-i}} e_{j_{s}}+x_{s}
$$

so that the $x_{s}, s \leq m_{j-i}$, are equally distributed vectors, and $\sum_{s=1}^{m_{j-i}} x_{s}$ has the same distribution as $y\left(m_{j-i+1}, \ldots m_{j}\right)$. It follows therefore from the induction hypothesis (32) (for $i-1$ ) that $\left\|x_{s}\right\| \leq C_{j-i+1} / m_{j-i}$, for $s=1,2 \ldots m_{j-1}$. Thus Lemma 2.8 is satisfied with $m=m_{j-i}, k=m_{j-i-1}, \varepsilon=\varepsilon_{j-i}$ and $C=C_{j-i+1}$, and we deduce that $\| y\left(m_{j-i}, m_{j-i+1}, \ldots, m_{j}\right) \leq\left(1+\varepsilon_{j-i}\right) C_{j-i+1}=C_{j-i}$, which implies (31). Moreover, the second part of the conclusion of Lemma 2.8 yields that if we write $y\left(m_{j-i}, m_{j-i+1}, \ldots, m_{j}\right)$ as sum of a block of $m_{j-i-1}$ equally distributed vectors $\tilde{y}_{1}<\tilde{y}_{2}<\ldots \tilde{y}_{m_{j-i-1}}$, we deduce that $\left\|\tilde{y}_{t}\right\| \leq\left(1+\varepsilon_{j-i}\right) C_{j-i+1} / m_{j-i-1}=C_{j-i} / m_{j-i-1}$, $t=1,2, \ldots, m_{j-i-1}$. Since the unit vector basis in $S$ is 1-unconditional this implies that $\left(y_{t}: 1 \leq t \leq m_{j-i-1}\right)$, with $y_{t}=m_{j-i-1} \tilde{y}_{t} / C_{j-i}$, for $t=1,2, \ldots, m_{j-i-1}$, is $C_{j-i}$-equivalent to the $\ell_{1}^{m_{j-i-1}}$ basis. Thus $y\left(m_{j-i}, m_{j-i+1}, \ldots, m_{j}\right) / C_{j-i}$, is an $\ell_{1}^{m_{j-i-1}}$ average up to the constant $1 / C_{j-i}$, in the way it is described by (32).

\section{Construction of a version of Gowers Maurey space}

To define the space $\mathcal{G} \mathcal{M}$, which will be a version of the space $G M$ introduced in [GM], we need to choose several objects. 
First, assume that $\bar{\varepsilon}=\left(\varepsilon_{n}\right)_{n \geq 0} \subset(0,1)$ satisfies the following standard conditions

$$
\varepsilon_{0}<\frac{f(2)-1}{2}, \varepsilon_{n} \leq 2^{-n} \text { and } \sum_{i>n} i^{2} \varepsilon_{i} \leq \frac{1}{10} \varepsilon_{n} \text {, for } n \in \mathbb{N} .
$$

Secondly, let $\mathbf{Q}$ be a countable set of elements of $c_{00}$, so that

$$
\left\{\sum_{i=1}^{l} a_{i} e_{i}: l \in \mathbb{N}, a_{i} \in \mathbb{Q}, \text { for } i=1,2, \ldots, l\right\} \cap[-1,1]^{\mathbb{N}} \subset \mathbf{Q} \subset c_{00} \cap[-1,1]^{\mathbb{N}},
$$

(35) if $x \in \mathbf{Q}$ and $E \subset \mathbb{N}$ is finite, then $E(x) \in \mathbf{Q}$,

(36) if $\left(x_{i}\right)_{i=1}^{l} \in \mathbf{Q}^{l}$ is a finite block sequence, then $\frac{1}{f(l)} \sum_{i=1}^{l} x_{i}$ and $\frac{1}{\sqrt{f(l)}} \sum_{i=1}^{l} x_{i}$ are in $\mathbf{Q}$.

Next we introduce a lacunary set $J \subset \mathbb{N}$. We write $J$ as an increasing sequence $\left\{j_{1}, j_{2}, \ldots\right\}$, and require the following four growth conditions

$$
\sum_{i>n} \frac{2}{j_{i}}<\frac{1}{f\left(j_{n}\right)} \text {, for all } n \in \mathbb{N},
$$

$\left(j_{i}\right)_{i=1}^{\infty}$ is admissible, and satisfies the conditions (25) and (26) imposed on $\left(m_{i}\right)_{j=1}^{\infty}$ in Lemma 2.9 (relative to the sequence $\left(\varepsilon_{n}\right)$ as chosen above).

In order to formulate the last condition on $J$, we first need to state an observation which is an easy consequence of James' blocking argument.

Lemma 3.1. For all $n \in \mathbb{N}$ and all $\varepsilon>0$ there is an $N=N(n, \varepsilon)$ so that the following holds:

Assume that $\left(E,\|\cdot\|_{E}\right)$ is a Banach space with a normalized and subsymmetric basis $\left(e_{i}\right)$, and there is a $c \in(0,1]$ so that for all $k \in \mathbb{N}$

$$
\left\|\sum_{j=1}^{k} e_{i}\right\|_{E} \geq c \frac{k}{f(k)} .
$$

Then, for all $\varepsilon>0$ and $n \in \mathbb{N}$, there is an $m \in[n, N(n, \varepsilon)]$ which is divisible by $n$, and there are $n$ subsets $A_{1}<A_{2}<\ldots A_{n}$ of $\{1,2, \ldots, m\}$, all of cardinality $m / n$, so that $\left(x_{i}: i=1,2, \ldots, n\right)$ is $c^{1 / n}(1-\varepsilon)$-equivalent to the $\ell_{1}^{n}$-unit vector basis, where

$$
x_{i}=\frac{\sum_{j \in A_{i}} e_{i}}{\left\|\sum_{j \in A_{i}} e_{j}\right\|} \text { for } i=1,2 \ldots, n .
$$

Our fourth condition on $J=\left\{j_{1}, j_{2}, \ldots\right\}$ can now be stated as follows (the first inequality being trivial):

$$
j_{s} \leq N\left(j_{s}, \varepsilon_{s}\right) \leq \frac{1}{2} \varepsilon_{s+1} f\left(j_{s+1}\right) .
$$


Finally we will need an injective function $\sigma$ from the collection of all finite sequences of elements of $\mathbf{Q}$ to the set $\left\{j_{2}, j_{4}, \ldots\right\}$ such that if $l \in \mathbb{N}, z_{1}^{*}, z_{2}^{*}, \ldots z_{l}^{*} \in \mathbf{Q}$ and $N=\max \left(\cup_{s=1}^{i} \operatorname{supp}\left(z_{s}^{*}\right)\right)$, then

$$
\varepsilon_{N} f\left(\sigma\left(z_{1}^{*}, \ldots, z_{i}^{*}\right)\right) \geq N .
$$

Depending on our choice of $\varepsilon, \mathbf{Q}, J$ and $\sigma$ we can now define recursively subsets $\mathcal{G M}_{m}^{*}$ in $c_{00} \cap[-1,1]^{\mathbb{N}}$, for each $m \in \mathbb{N}_{0}$, which will serve as a set of normalizing functionals of $\mathcal{G} \mathcal{M}$.

Let

$$
\mathcal{G} \mathcal{M}_{0}^{*}=\left\{\lambda e_{n}^{*}: n \in \mathbb{N},|\lambda| \leq 1\right\} .
$$

Assume that $\mathcal{G} \mathcal{M}_{m}^{*}$ has been defined for some $m \in \mathbb{N}_{0}$. Then $\mathcal{G} \mathcal{M}_{m+1}^{*}$ is the set of all functionals of the form $E\left(z^{*}\right)$ where $E \subseteq \mathbb{N}$ is an interval and $z^{*}$ has one of the following three forms (41), (42) or (43):

$$
z^{*}=\sum_{i=1}^{l} \alpha_{i} z_{i}^{*}
$$

where $\sum_{i=1}^{l}\left|\alpha_{i}\right| \leq 1$ and $z_{i}^{*} \in \mathcal{G} \mathcal{M}_{m}^{*}$ for $i=1, \ldots, k$.

$$
z^{*}=\frac{1}{f(l)} \sum_{i=1}^{l} z_{i}^{*}
$$

where $z_{i}^{*} \in \mathcal{G} \mathcal{M}_{m}^{*}$ for $i=1, \ldots, l$, and $z_{1}^{*}<\cdots<z_{l}^{*}$.

$$
z^{*}=\frac{1}{\sqrt{f(k)}} \sum_{i=1}^{k} z_{i}^{*} \text { and } z_{i}^{*}=\frac{1}{f\left(n_{i}\right)} \sum_{j=1}^{n_{i}} z_{i, j}^{*}
$$

where

a) $z_{1,1}^{*}<\cdots<z_{1, n_{1}}^{*}<z_{2,1}^{*}<\cdots<z_{l, n_{l}}^{*}$,

b) $z_{i, j}^{*} \in \mathcal{G M}_{m}^{*} \cap \mathbf{Q}$, for $1 \leq i \leq k$ and $1 \leq j \leq n_{i}$ (and thus $z_{i}^{*} \in \mathbf{Q}$, for $i=1,2 \ldots k)$, and

c) $n_{1}=j_{2 k^{\prime}}$, for some $k^{\prime} \geq k$, and $n_{i+1}=\sigma\left(z_{1}^{*}, \ldots, z_{i}^{*}\right)$, for $i=1, \ldots, k-1$.

Finally, the norm of $\mathcal{G} \mathcal{M}$ is defined by

$$
\|x\|_{\mathcal{G M}}=\sup \left\{z^{*}(x): z^{*} \in \cup_{m=0}^{\infty} \mathcal{G} \mathcal{M}_{m}^{*}\right\} .
$$

Remark. There are two main technical differences between the original space $G M$ defined in $[\mathrm{GM}]$ and the space $\mathcal{G} \mathcal{M}$ defined here:

(1) we allow in (43) $k$ to take any value in $\mathbb{N}$, while in [GM] $k$ had to be chosen out of the very lacunary set $\left\{j_{2 s+1}, s \in \mathbb{N}\right\}$ and $\sigma$ in GM could only take values in $\left\{j_{2 s}: s \in \mathbb{N}\right\}$.

(2) in (43) we allow that $n_{1}$ is of the form $n_{1}=j_{2 k^{\prime}}$, with $k^{\prime} \geq k$, while in [GM], it is required that $k^{\prime}=k$. 
The point is that it is not enough to use the coding procedure of [GM] to obtain as they do, given $\epsilon>0$, some $k$ and two intertwined finite sequences $u_{1}<v_{1}<\cdots<u_{k}<v_{k}$ such that $\left\|\sum_{i=1}^{k}\left(u_{i}-v_{i}\right)\right\| \leq \epsilon\left\|\sum_{i=1}^{k}\left(u_{i}+v_{i}\right)\right\|$. To deduce estimates about spreading models, we need this to be valid for any $k$ large enough and for any initial vector $u_{1}$ far enough along the basis.

The proof that our construction still does not contain an unconditional basis becomes therefore a bit harder. Nevertheless the main ideas of the proof stay the same.

Notation. For $m \in \mathbb{N}$, and if $X$ is a Banach space with a normalized basis $\left(e_{i}\right)$ (we will use this notation for $S$ as well as for $\mathcal{G M}$ ).

$$
A_{m}^{*}(X)=\left\{\frac{1}{f(l)} \sum_{i=1}^{l} x_{i}^{*}: x_{1}^{*}<x_{2}^{*}<\ldots x_{l}^{*} \text { in } B_{X^{*}} \cap c_{00}\right\} .
$$

Note that $A_{m}^{*}(\mathcal{G M}) \subset B_{\mathcal{G M}^{*}}$ and $A_{m}^{*}(S) \subset B_{S^{*}}$.

We define for $x \in X$ and $m \in \mathbb{N}$

$$
\|x\|_{m}=\sup _{x^{*} \in A_{m}^{*}}\left|x^{*}(x)\right|=\max _{E_{1}<E_{2}<\ldots E_{m}} \frac{1}{f(m)} \sum_{i=1}^{m}\left\|E_{i}(x)\right\| .
$$

and observe that

$$
\frac{1}{f(m)}\|x\|_{S} \leq\|x\|_{m} \leq\|x\|_{S} \leq\|x\|_{\mathcal{G M}}
$$

For $k \in \mathbb{N}$ we also define

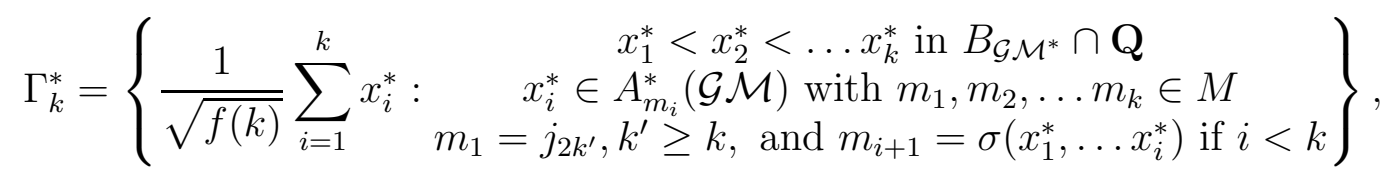

and put for $x \in \mathcal{G M}$

$$
\|x\|_{G_{m}^{*}}=\sup _{x^{*} \in \Gamma_{m}^{*}}\left|x^{*}(x)\right| .
$$

\section{Some technical observations Concerning the SPACE $\mathcal{G} \mathcal{M}$}

In this section we prove several properties of the space $\mathcal{G} \mathcal{M}$, as defined in the previous section. In particular we will conclude that also this version does not contain any unconditional basic sequences. In this section we will abbreviate $\|\cdot\|_{\mathcal{G M}}$ by $\|\cdot\|$.

The following observation follows from James' blocking argument (See Lemma 3.1).

Lemma 4.1. The space $\ell_{1}$ is finitely block represented in every infinite dimensional block subspace of $\mathcal{G} \mathcal{M}$.

The next Lemma is easy to show (c.f. [Sch1] or [GM])

Lemma 4.2 (Action of $\|\cdot\|_{l}$ on $\ell_{1}^{+}$averages). Assume that $x \in B_{\mathcal{G M}}$ is an $\ell_{1}^{n}$-average and $l \in \mathbb{N}$. Then

$$
\|x\|_{l} \leq \frac{1}{f(l)}\left(1+\frac{l}{n}\right)
$$


Definition 4.3. (Rapidly Increasing Sequences) We call a block sequence $\left(x_{n}\right) \subset$ $B_{\mathcal{G M}}$ rapidly increasing sequence of constant $c$ or $c$-RIS, with $c \in(0,1]$ if the following two conditions (45) and (46) are satisfied (recall that the sequence $\varepsilon_{n}$ is given by (33)):

For $n \in \mathbb{N}, x_{n}$ is an $\ell_{1}^{k_{n}}$-average of constant $c$, if $c<1$, or of constant

$1 /\left(1+\varepsilon_{n}\right)$, if $c=1$, and the following two inequalities are satisfied:

$\max \left(\frac{2 n}{f\left(k_{n}\right)}, \frac{f\left(k_{n}\right)}{k_{n}}\right)<\varepsilon_{n}^{2}$ and $f\left(\varepsilon_{n} \sqrt{k_{n}}\right) \geq \frac{1}{\varepsilon_{n}^{2}} \max \operatorname{supp}\left(x_{n-1}\right)$, if $n \geq 2$,

$\left(x_{n}\right)$ has a spreading model $E$ with a 1-unconditional and seminormalized

basis $\left(e_{i}\right)$ and for $l \in \mathbb{N}$ and $\left(a_{i}\right)_{i=1}^{l} \subset \mathbb{R}$ and $l \leq n_{1}<n_{2}<\ldots n_{l}$ in $\mathbb{N}$

$$
\frac{1}{1+\varepsilon_{l}}\left\|\sum_{i=1}^{l} a_{i} e_{i}\right\|_{E} \leq\left\|\sum_{i=1}^{l} a_{i} x_{n_{i}}\right\|_{E} \leq\left(1+\varepsilon_{l}\right)\left\|\sum_{i=1}^{l} a_{i} e_{i}\right\|_{E} .
$$

We say that a sequence $\left(x_{n}\right)$ is an RIS, if it is an $c$-RIS for some constant $c$. If $c=1$, we say that $\left(x_{n}\right)$ is an asymptotically isometric RIS.

We note that from Lemma 4.1 it follows immediately that any infinite dimensional block subspace $Y$ of $\mathcal{G M}$ contains an asymptotically isometric RIS.

Remark. Let $\left(x_{n}\right)$ be a $c$-RIS, and $(E,\|\cdot\|)$ be the spreading model of $\left(x_{n}\right)$. Define for $l \in \mathbb{N}$

$$
g(l)=\frac{l}{\left\|\sum_{i=1}^{l} e_{i}\right\|_{E}}=\lim _{n_{1}<n_{2}<\ldots n_{l}} \frac{l}{\left\|\sum_{i=1}^{l} x_{n_{i}}\right\|} .
$$

From the construction of $\mathcal{G M}$ it follows that

$$
g(l) \leq f(l) / c \text { for all } l \in \mathbb{N}
$$

in particular the spreading model $E$ of $\left(x_{n}\right)$ satisfies the conditions of Lemma 3.1.

It follows therefore that for $n \in \mathbb{N}$ and $\varepsilon>0$ we can choose an appropriate $m \in$ $[n, N(n, \varepsilon)]$ and $m$ elements from $\left(x_{j}\right)$ so that their sum is up to a scalar multiple, which is as close to $\frac{g(m)}{m}$, as we wish, an $\ell_{1}^{n}$ average of constant $c-\varepsilon$.

Thus it is justified to introduce the following notion of Special Rapidly Increasing Sequences.

Definition 4.4. (Special Rapidly Increasing Sequences) A block sequence $\left(x_{n}\right)$ in $B_{G M}$ is called a Special Rapidly Increasing Sequence of constant $c$, with $c \in(0,1]$, or $c$-SRIS, if there is a $c$-RIS $\left(\tilde{x}_{n}\right) \subset B_{G M}$, so that for each $n \in \mathbb{N}$ there is $\tilde{p}=\tilde{p}(n) \in P$, $\tilde{p} \geq n, \tilde{m}(n) \in\left[j_{\tilde{p}(n)}, N\left(j_{\tilde{p}(n)}, \varepsilon_{\tilde{p}(n)}\right)\right]$ (here $N(\cdot, \cdot)$ is chosen as in Lemma 3.1) and natural numbers $\tilde{m}(n) \leq \tilde{s}(n, 1)<\tilde{s}(n, 2)<\ldots \tilde{s}(n, \tilde{m}(n))$, so that

$$
x_{n}=\frac{\tilde{g}(\tilde{m}(n))}{\tilde{m}(n)} \sum_{r=1}^{\tilde{m}(n)} \tilde{x}_{\tilde{s}(n, r)},
$$


where

$$
\tilde{g}(l)=\frac{l}{\left\|\sum_{i=1}^{l} \tilde{e}_{i}\right\|_{\tilde{E}}}=\lim _{n_{1}<n_{2}<\ldots n_{l}} \frac{l}{\left\|\sum_{i=1}^{l} \tilde{x}_{n_{i}}\right\|_{\tilde{E}}}
$$

and $\tilde{E}$ is the spreading model of $\left(\tilde{x}_{n}\right)$ with semi normalized 1-unconditional basis $\left(\tilde{e}_{n}\right)$,

$$
\begin{aligned}
& x_{n} \text { is an } \ell_{1}^{j_{\tilde{p}(n)}} \text {-average of constant } c \text {, if } c<1 \text {, or } 1 /\left(1+\varepsilon_{\tilde{p}(n)}\right) \text {, if } c=1, \\
& \left(x_{n}\right) \text { is an RIS of constant } c, \text { with } k_{n}=j_{\tilde{p}(n)} \text { in condition (45). }
\end{aligned}
$$

Remark. From the remark before Definition 4.4 it follows that every block subspace contains special rapidly increasing sequences. The point of Definition 4.4 is that we may regard the $x_{n}$ at the same time as $\ell_{1}^{k_{n}}$-averages for fast increasing $k_{n}$, but also, up to some factor, sums of elements of an RIS. We shall use this to prove that SRIS generate spreading models equivalent to the unit vector basis of $S$. Note that every normalized block basis of $\mathcal{G} \mathcal{M}$ dominates the unit basis of $S$.

Lemma 4.5 (Action of $A_{l}^{*}$ on sums of elements of an RIS). Assume that $\left(x_{n}\right)$ is a c-RIS, $c \in(0,1]$. Let $m \leq n_{1}<n_{2}<\ldots n_{m}$ be in $\mathbb{N}$ and $\left(a_{i}\right)_{i=1}^{m} \in \mathbb{R}^{m}$. Put $y=\sum_{i=1}^{m} a_{i} x_{n_{i}}$.

a) If $f(l) \leq 2 m / \varepsilon_{n_{1}}$ then there are numbers $l_{1}$ and $l_{2}$ in $\mathbb{N}$, with $l_{1}+l_{2} \leq$ $\min (2 l, m)$, intervals $I_{1}<I_{2}<\ldots<I_{l_{1}}$ in $\{1,2 \ldots m\}$, so that $l_{2}=\# I_{0}$, with $I_{0}=\{1,2 \ldots\} \backslash \bigcup_{j=1}^{l_{1}} I_{j}$ and

$$
\|y\|_{\ell} \leq \frac{1}{f(l)}\left[\sum_{j=1}^{l_{1}}\left\|\sum_{s \in I_{j}} a_{s} x_{n_{s}}\right\|+\sum_{s \in I_{0}}\left|a_{s}\right| \frac{1+2 \varepsilon_{n_{s}}}{c}\left\|x_{n_{s}}\right\|\right],
$$

b) If $f(l)>2 m / \varepsilon$, for some $\varepsilon \in\left[\varepsilon_{n_{1}}, 1\right)$, then

$$
\|y\|_{l} \leq \max _{i \leq m}\left|a_{i}\right|\left[2 \varepsilon+\max _{i=1,2 \ldots m}\left\|x_{n_{i}}\right\|_{l}\right] \leq \max _{i \leq m}\left|a_{i}\right|[2 \varepsilon+1]
$$

Proof. We put $z_{s}=x_{n_{s}}$ for $s=1,2 \ldots m$. In order to prove (a) we choose finite intervals $E_{1}<E_{2}<\ldots E_{l}$ of $\mathbb{N}$, so that

$$
\|y\|_{l}=\frac{1}{f(l)} \sum_{t=1}^{l}\left\|E_{t}(y)\right\| .
$$

Without loss of generality we can assume that

$$
\bigcup_{t=1}^{l} E_{t}=\operatorname{ran}(y)=\left[\min \operatorname{supp}\left(z_{1}\right), \max \operatorname{supp}\left(z_{m}\right)\right]
$$

For $t=1,2 \ldots l$ we divide $E_{t}$ in three intervals $E_{t}^{(1)}, E_{t}^{(2)}, E_{t}^{(3)}$ (some of them possibly empty) as follows: we let, if it exists, $m(t)$ be the unique number in $\{1,2, \ldots m\}$ so that $\min \operatorname{ran}\left(z_{m(t)}\right)<\min E_{t} \leq \max \operatorname{ran}\left(z_{(m(t)}\right)$ and put

$$
E_{t}^{(1)}=E_{t} \cap\left[1, \max \operatorname{ran}\left(z_{m(t)}\right)\right]
$$


If $m(t)$ does not exists we let $E_{t}^{(1)}=\emptyset$. Then we let $m^{\prime}(t)$ be the unique number $m^{\prime}(t)$, if it exists, so that $\min \operatorname{ran}\left(z_{m^{\prime}(t)}\right)<\max E_{t} \leq \max \operatorname{ran}\left(z_{(m(t)}\right)$, and put

$$
E_{t}^{(3)}=\left(E_{t} \cap\left[\min \operatorname{ran}\left(z_{m^{\prime}(t)}\right), \infty\right)\right) \backslash E_{t}^{(1)} .
$$

If $m^{\prime}(t)$ does not exists we let $E_{t}^{(1)}=\emptyset$. Finally we let $E_{t}^{(2)} \backslash\left(E_{t}^{(1)} \cup E_{t}^{(3)}\right)$. Let $\tilde{\mathcal{E}}$ be the non empty elements of $\left\{E_{t}^{(1)}, E_{t}^{(2)}, E_{t}^{(3)}: t \leq l\right\}$ and $\tilde{l}$ the cardinality of $\tilde{\mathcal{E}}$.

We note that $\tilde{\mathcal{E}}$ consists of pairwise disjoint intervals which can be ordered into $\tilde{E}_{1}<\tilde{E}_{2}<\ldots \tilde{E}_{\tilde{l}}$, and that for any $i \leq m$ and any $j \leq \tilde{l}$, either $\tilde{E}_{j}$ contains $\operatorname{ran}\left(z_{i}\right)$, or is contained in $\operatorname{ran}\left(z_{i}\right)$, or $\tilde{E}_{j}$ and $\operatorname{ran}\left(z_{i}\right)$ are disjoint.

For $s \in\{1,2, \ldots, m\}$ we deduce from our condition on $f(l)$ and (45) that

$$
\frac{l}{k_{n_{s}}} \leq \frac{f(l)}{f\left(k_{n_{s}}\right)} \leq \frac{2 m}{\varepsilon_{n_{1}} f\left(k_{n_{s}}\right)} \leq \frac{2 n_{s}}{\varepsilon_{n_{1}} f\left(k_{n_{s}}\right)} \leq \frac{\varepsilon_{n_{s}}^{2}}{\varepsilon_{n_{1}}} \leq \varepsilon_{n_{s}} .
$$

We let

$$
I_{0}=\left\{s=1,2 \ldots, m: \#\left\{t: \tilde{E}_{t} \subset \operatorname{ran}\left(x_{s}\right)\right\} \geq 2\right\},
$$

Lemma 4.2 yields that for every $s \in I_{0}$

$$
\sum_{t, \tilde{E}_{t} \subset \operatorname{ran}\left(z_{s}\right)}\left\|\tilde{E}_{t}\left(z_{s}\right)\right\| \leq 1+\frac{l}{k_{n_{s}}} \leq 1+\varepsilon_{n_{s}} \leq \frac{\left\|z_{s}\right\|+2 \varepsilon_{n_{s}}}{c} .
$$

We reorder the set $\tilde{\mathcal{E}}^{\prime}$ of all sets $\tilde{E}_{t}, t \in\{1,2 \ldots \tilde{l}\}$, which contain the range of at least one $x_{n_{s}}$, into $E_{1}^{\prime}<\ldots E_{l_{1}}^{\prime}$ and we define $t=1, \ldots l_{1}$

$$
I_{t}=\left\{s \in\{1,2 \ldots m\}: \operatorname{ran}\left(x_{s}\right) \subset \tilde{E}_{t}\right\},
$$

and conclude that $l_{1}+l_{2} \leq \min (m, 2 l)$, where $l_{2}=\# I_{0}$, and

$$
\begin{aligned}
\frac{1}{f(l)} \sum_{t=1}^{l}\left\|E_{i}(y)\right\| & \leq \frac{1}{f(l)} \sum_{t=1}^{\tilde{l}}\left\|\tilde{E}_{t}(y)\right\| \\
& =\frac{1}{f(l)}\left[\sum_{t=1}\left\|\sum_{s \in I_{t}} a_{s} z_{s}\right\|+\sum_{s \in I_{0}}\left|a_{s}\right| \sum_{t=1}^{\tilde{l}}\left\|\tilde{E}_{t}\left(z_{s}\right)\right\|\right] \\
& \leq \frac{1}{f(l)}\left[\sum_{t=1}\left\|\sum_{s \in I_{t}} a_{s} z_{s}\right\|+\sum_{s \in I_{0}}\left|a_{s}\right| \frac{\left\|z_{s}\right\|+\varepsilon_{n_{s}}}{c}\right]
\end{aligned}
$$

which implies (a).

In order to prove our claim (b) let $\varepsilon \in\left[\varepsilon_{n_{1}}, 1\right]$ and define

$$
i_{0}=\max \left\{i=1,2 \ldots k: \max \left(\operatorname{supp}\left(z_{i-1}\right)\right)<f(l) \varepsilon\right\}
$$

$\left(\right.$ with $\left.\max \left(\operatorname{supp}\left(z_{0}\right)\right):=0\right)$.

Then by (45) it follows for $i \in\left\{i_{0}+1, i_{0}+2, \ldots, m\right\}$ that

$$
f\left(k_{n_{i}}\right)>\frac{1}{\varepsilon_{n_{i}}} \max \operatorname{supp}\left(z_{i-1}\right) \geq \frac{1}{\varepsilon} \max \operatorname{supp}\left(z_{i_{0}}\right) \geq f(l)
$$


and thus,

$$
\begin{aligned}
\left\|\sum_{i=1}^{m} a_{i} z_{i}\right\|_{l} & \leq \frac{1}{f(l)} \sum_{i=1}^{i_{0}-1}\left|a_{i}\right|\left\|z_{i}\right\|_{l}+\left|a_{i_{0}}\right|\left\|z_{i_{0}}\right\|_{l}+\frac{1}{f(l)} \sum_{i=i_{0}+1}^{m}\left|a_{i}\right|\left\|z_{i}\right\|_{l} \\
& \leq \max _{i \leq m}\left|a_{i}\right|\left[\frac{\max \operatorname{supp}\left(z_{i_{0}-1}\right)}{f(l)}+\left\|z_{i_{0}}\right\|_{l}+\frac{1}{f(l)} \sum_{i=i_{0}+1}^{m}\left(1+\frac{l}{k_{n_{i}}}\right)\right]
\end{aligned}
$$

(by Lemma 4.2)

$$
\leq \max _{i \leq m}\left|a_{i}\right|\left[\varepsilon+\left\|z_{i_{0}}\right\|_{l}+\frac{2 m}{f(l)}\right]<\max _{i \leq m}\left|a_{i}\right|\left[2 \varepsilon+\left\|z_{i_{0}}\right\|_{l}\right]
$$

which proves part (b).

Lemma 4.6 (Action of $\Gamma_{k}^{*}$ on sums of elements of an RIS). Assume that $\left(x_{n}\right)$ is a block-sequence in $\mathcal{G M}, c \in(0,1]$, and let $z^{*} \in \Gamma_{k}^{*}$. Let $m \leq n_{1}<n_{2}<\ldots n_{m}$ be in $\mathbb{N}$ and $\left(a_{i}\right)_{i=1}^{m} \in \mathbb{R}^{m}$. Put $y=\sum_{i=1}^{m} a_{i} x_{n_{i}}$.

a) If $\left(x_{n}\right)$ is a c-RIS, then

$$
\begin{gathered}
\left|z^{*}(y)\right| \leq \frac{\left|z_{t_{0}}^{*}(y)\right|}{\sqrt{f(k)}}+\frac{\max _{s \leq m}\left|a_{s}\right|}{\sqrt{f(k)}}\left[1+2 m \varepsilon_{n_{1}}+\sum_{t \in T_{0}} \max _{s \in S_{t}}\left\|x_{n_{s}}\right\|_{l_{t}}\right] \\
\quad+\frac{1}{\sqrt{f(k)}} \sum_{s=s_{0}+1}^{m}\left|a_{s}\right|\left|\sum_{t \in T_{s}} z_{t}^{*}\left(x_{n_{s}}\right)\right|
\end{gathered}
$$

where $t_{0} \in\{1,2 \ldots k\}, T_{s}, \subset\left\{t_{0}+1, t_{0}+2 \ldots k\right\}, s=0,1,2 \ldots m$ are defined as follows:

$t_{0}=\min \left\{t=1, \ldots k: z_{t}^{*}(y) \neq 0\right\}$,

(we assume that $t_{0}$ exists, otherwise $z^{*}(y)=0$ )

$T_{s}=\left\{t=t_{0}+1, \ldots k: \operatorname{supp}\left(z_{t}^{*}\right) \subset\left[\min \operatorname{ran}\left(z_{s}\right), \min \operatorname{ran}\left(z_{s+1}\right)\right)\right\}$, if $s=1, \ldots k$

$\left(\right.$ with $\left.\min \operatorname{ran}\left(z_{m+1}\right):=\infty\right)$

$$
T_{0}=\left\{t_{0}+1, \ldots k\right\} \backslash \bigcup_{s=1}^{k} T_{s} \text {. }
$$

b) If $\left(x_{n}\right)$ s a c-SRIS, then

$$
\left|z^{*}(y)\right| \leq \frac{\left|z_{t_{0}}^{*}(y)\right|}{\sqrt{f(k)}}+\frac{\max _{s \leq m}\left|a_{s}\right|}{\sqrt{f(k)}} \min (m, k) \max _{s \leq m, t_{0}<t \leq k}\left\|x_{s}\right\|_{l_{t}}+2 \max _{s \leq m}\left|a_{s}\right| .
$$

Proof. We first assume that $\left(x_{n}\right)$ is only a $c$-RIS. For $m \leq n_{1}<n_{2}<\ldots n_{s}$ in $\mathbb{N}$ and $\left(a_{s}\right)_{s=1}^{m} \subset \mathbb{R} \backslash\{0\}$ we put

$$
y=\sum_{s=1}^{m} a_{s} x_{n_{s}}
$$


Secondly let $k \in \mathbb{N}$ and $z^{*} \in \Gamma_{k}^{*}$. We write $z^{*}$ as

$$
z^{*}=\frac{1}{\sqrt{f(k)}} \sum_{t=1}^{k} z_{t}^{*} \in \Gamma_{k}^{*},
$$

with $z_{1}^{*} \in A_{l_{1}}^{*}$, and $l_{1}=j_{2 k^{\prime}}$, for some $k^{\prime} \geq k$, and $z_{i}^{*} \in A_{l_{i}}^{*}$, with $l_{i}=\sigma\left(z_{1}^{*}, z_{2}^{*}, \ldots, z_{i-1}^{*}\right)$, for $i=2, \ldots k$. We note that for $t_{0}$ as defined in the statement it follows that

$$
\max \left(\operatorname{supp}\left(z_{t_{0}}^{*}\right)\right) \geq \min \left(\operatorname{supp}\left(x_{n_{1}}\right)\right) .
$$

We abbreviate $z_{s}=a_{s} x_{n_{s}}$, for $s=1,2 \ldots m$. For $t \in T_{0}$ let

$$
S_{t}=\left\{s \in\{1,2 \ldots m\}: \operatorname{ran}\left(z_{t}^{*}\right) \cap \operatorname{ran}\left(z_{s}\right) \neq \emptyset\right\} .
$$

Note that $S_{t}$ is an interval in $\{1,2, \ldots m\}$ and that each $s \in\{1,2 \ldots, m\}$ maybe element in at most two of the sets $S_{t}, t \in T_{0}$. Using these notations, we can now write $z^{*}(y)$ as

$$
z^{*}(y)=\frac{1}{\sqrt{f(k)}} z_{t_{0}}^{*}(y)+\frac{1}{\sqrt{f(k)}} \sum_{t \in T_{0}} \sum_{s \in S_{t}} z_{t}^{*}\left(z_{s}\right)+\frac{1}{\sqrt{f(k)}} \sum_{s=1}^{m} \sum_{t \in T_{s}} z_{t}^{*}\left(z_{s}\right) .
$$

In order to estimate the second term in (56) we first deduce from (40) and the trivial estimate $\min \operatorname{supp}\left(z_{1}\right)>2 n_{1}$, that for $t \in T_{0}$

$$
f\left(l_{t}\right) \geq \frac{\max \operatorname{supp}\left(z_{t_{0}}^{*}\right)}{\varepsilon_{\max \sup \left(z_{t_{0}}^{*}\right)}} \geq \frac{\min \operatorname{supp}\left(z_{1}\right)}{\varepsilon_{n_{1}}}>\frac{2 n_{1}}{\varepsilon_{n_{1}}} \geq \frac{2 m}{\varepsilon_{n_{1}}},
$$

and Lemma 4.5 (b) yields therefore that

$$
\left|z^{*}\left(\sum_{s \in S_{t}} z_{s}\right)\right| \leq \max _{s \in S_{t}}\left|a_{i}\right|\left[2 \varepsilon_{n_{1}}+\max _{s \in S_{t}}\left\|x_{n_{s}}\right\|_{l_{t}}\right] \text {, whenever } t \in T_{0} .
$$

In order to estimate the third term in (56) we define

$$
s_{0}=\min \left\{s=1,2 \ldots m: \max \operatorname{supp}\left(z_{s-1}\right)<\varepsilon_{n_{1}} \sqrt{f(k)}\right\}
$$

with the usual convention that $\max \operatorname{supp}\left(z_{0}\right)=0$. We first note that if $s_{0} \geq 2$

$$
\frac{1}{\sqrt{f(k)}} \sum_{s=1}^{s_{0}-1} \sum_{t \in T_{s}}\left|z_{t}^{*}\left(z_{s}\right)\right| \leq \frac{1}{\sqrt{f(k)}} \max \operatorname{supp}\left(z_{s_{0}-1}\right) \max _{s \leq m}\left|a_{s}\right| \leq \varepsilon_{n_{1}} \max _{s \leq m}\left|a_{s}\right| .
$$

Secondly, if $T_{s_{0}} \neq \emptyset$ we let

$$
I=\left[\min \bigcup_{t \in T_{s_{0}}} \operatorname{ran}\left(z_{t}^{*}\right), \max \bigcup_{t \in T_{s_{0}}} \operatorname{ran}\left(z_{t}^{*}\right)\right],
$$

and deduce that

$$
\frac{1}{\sqrt{f(k)}}\left|\sum_{t \in T_{s_{0}}} z_{t}^{*}\left(z_{s_{0}}\right)\right|=\left|I\left(z^{*}\right)\left(z_{s_{0}}\right)\right| \leq\left|a_{s_{0}}\right| \cdot\left\|x_{n_{s_{0}}}\right\| \leq \max _{s \leq m}\left|a_{s}\right| .
$$


Adding up the estimates obtained in (59) and (160) and inserting them into (56), we obtain

$$
\begin{aligned}
&\left|z^{*}(y)\right| \leq \frac{\left|z_{t_{0}}^{*}(y)\right|}{\sqrt{f(k)}}+\frac{1}{\sqrt{f(k)}} \sum_{t \in T_{0}} \max _{s \in S_{t}}\left|a_{s}\right|\left[\max _{s \in S_{t}}\left\|x_{n_{s}}\right\|_{l_{t}}+2 \varepsilon_{n_{1}}\right] \\
& \quad+\max _{s \leq m}\left|a_{s}\right|+\frac{1}{\sqrt{f(k)}} \sum_{s=s_{0}+1}^{m}\left|a_{s}\right|\left|\sum_{t \in T_{s}} z_{t}^{*}\left(x_{n_{s}}\right)\right| \\
& \leq \frac{\left|z_{t_{0}}^{*}(y)\right|}{\sqrt{f(k)}}+\frac{\max _{s \leq m}\left|a_{s}\right|}{\sqrt{f(k)}}\left[1+2 m \varepsilon_{n_{1}}+\sum_{t \in T_{0}} \max _{s \in S_{t}}\left\|x_{n_{s}}\right\|_{l_{t}}\right] \\
&+\frac{1}{\sqrt{f(k)}} \sum_{s=s_{0}+1}^{m}\left|a_{s}\right|\left|\sum_{t \in T_{s}} z_{t}^{*}\left(x_{n_{s}}\right)\right|
\end{aligned}
$$

which proves (54).

In order to prove part (b) we now assume that $\left(x_{n}\right)$ is an SRIS of constant $c$, and want for $s=s_{0}+1, \ldots m$, with $T_{s} \neq \emptyset$, to find an upper estimate for

$$
\frac{1}{\sqrt{f(k)}}\left|\sum_{t \in T_{s}} z_{t}^{*}\left(x_{n_{s}}\right)\right| \text {. }
$$

Thus, we assume that there is an RIS $\left(\tilde{x}_{n}\right) \subset B_{\mathcal{G M}}$ of constant $c$, and for each $n \in \mathbb{N}$ numbers $\tilde{p}(n) \in \mathbb{N}, \tilde{p}(n) \geq n, \tilde{m}(n) \in\left[j_{\tilde{p}(n)}, N\left(j_{\tilde{p}(n)}, \varepsilon_{\tilde{p}(n)}\right]\right.$ and $\tilde{m}(n) \leq \tilde{s}_{1}(n)<$ $s_{2}(n)<\ldots \tilde{s}_{\tilde{m}(n)}(n)$ so that

$$
x_{n}=\frac{\tilde{g}(\tilde{m}(n))}{\tilde{m}(n)} \sum_{r=1}^{\tilde{m}(n)} \tilde{x}_{\tilde{s}_{r}(n)},
$$

where $\tilde{g}:[1, \infty) \rightarrow[1, \infty)$ is an increasing function with $\tilde{g}(\xi) \leq f(\xi) / c, \xi \geq 1$ and so that $k_{n}=j_{\tilde{p}(n)}\left(\right.$ thus $x_{n}$ is an $\ell_{1}^{\tilde{p}(n)}$-average of constant $c$ ).

We fix $s=s_{0}+1, s_{+} 2, \ldots m$, with $T_{s} \neq \emptyset$, and apply now our estimate (61) to $x_{n_{s}}=\frac{\tilde{g}\left(\tilde{m}\left(n_{s}\right)\right)}{\tilde{m}\left(n_{s}\right)} \sum_{r=1}^{\tilde{m}\left(n_{s}\right)} \tilde{x}_{\tilde{s}_{r}\left(n_{s}\right)}$ instead of $y$ and to $\tilde{z}^{*}=\frac{1}{\sqrt{f(k)}} \sum_{t \in T_{s}} z_{t}^{*}$, instead of $z^{*}$. Strictly speaking $\tilde{z}^{*}$ is not in $\Gamma_{k}^{*}$, but it is of the form $I\left(z^{*}\right)$, where $I \subset \mathbb{N}$ is an interval, and it is easy to see that it satisfies the same estimates (61). From the definition of $s_{0}$ and by the second condition on $k_{n_{s}}$ in (46) we deduce that

$$
\sqrt{f(k)}<\frac{1}{\varepsilon_{n_{1}}} \operatorname{supp}\left(z_{s-1}\right)<\sqrt{f\left(\varepsilon_{n_{s}} k_{n_{s}}^{1 / 2}\right)}
$$

and thus

$$
k \leq \varepsilon_{n_{s}} k_{n_{s}}^{1 / 2}=\varepsilon_{n_{s}} j_{\tilde{p}\left(n_{s}\right)}^{1 / 2} \leq \varepsilon_{n_{s}} \tilde{m}_{s}^{1 / 2}\left(n_{s}\right)
$$

which yields

$$
\frac{k \tilde{g}\left(\tilde{m}\left(n_{s}\right)\right)}{\tilde{m}\left(n_{s}\right)} \leq \varepsilon_{n_{s}} \frac{\tilde{g}\left(\tilde{m}\left(n_{s}\right)\right)}{\tilde{m}^{1 / 2}\left(n_{s}\right)} \leq \varepsilon_{n_{s}} .
$$


Put $t_{s}=\min T_{s}$ (which takes the role of $t_{0}$ ). If we apply (61) to $x_{n_{s}}$, the sets $T_{0}$ and $T_{s}$ will be replaced by sets $\tilde{T}_{0}$ and $\tilde{T}_{\tilde{s}}, \tilde{s} \leq \tilde{m}\left(n_{s}\right)$, for which we know (and only will need to know) that

$$
\# \tilde{T}_{0} \leq k \text { and } \sum_{\tilde{s}=1}^{\tilde{m}\left(n_{s}\right)} \# \tilde{T}_{\tilde{s}} \leq k .
$$

Using also the estimate $m \varepsilon_{n_{1}}+\left\|\tilde{x}_{\tilde{s}_{r}\left(n_{s}\right)}\right\|+1 \leq 3$, we obtain from (61), applied to $\tilde{z}^{*}\left(x_{n_{s}}\right)$, that

$$
\left|\tilde{z}^{*}\left(x_{n_{s}}\right)\right| \leq \frac{\mid z_{t_{s}}^{*}\left(x_{n_{s}} \mid\right.}{\sqrt{f(k)}}+\frac{\tilde{g}(\tilde{m})}{\tilde{m}} \frac{3 k}{\sqrt{f(k)}}+\frac{\tilde{g}(\tilde{m})}{\tilde{m}} \frac{k}{\sqrt{f(k)}} \leq \frac{\left|z_{t_{s}}^{*}\left(x_{n_{s}}\right)\right|}{\sqrt{f(k)}}+4 \varepsilon_{n_{s}} .
$$

Inserting this estimate back into (61) for $\left|z^{*}(y)\right|$ we get

$$
\begin{aligned}
\left|\tilde{z}^{*}(y)\right| \leq & \frac{\left|z_{t_{0}}^{*}(y)\right|}{\sqrt{f(k)}}+\frac{\max _{s \leq m}\left|a_{s}\right|}{\sqrt{f(k)}}\left[1+2 m \varepsilon_{n_{1}}+\sum_{t \in T_{0}} \max _{s \in S_{t}}\left\|x_{n_{s}}\right\|_{l_{t}}\right] \\
& \quad+\frac{1}{\sqrt{f(k)}} \sum_{s=1, T_{s} \neq \emptyset}^{m}\left|a_{s}\right|\left[\mid z_{t_{s}}^{*}\left(x_{n_{s}}\right)+4 \varepsilon_{n_{s}}\right] \\
\leq & \frac{\left|z_{t_{0}}^{*}(y)\right|}{\sqrt{f(k)}}+\frac{\max _{s \leq m}\left|a_{s}\right|}{\sqrt{f(k)}}\left[\sum_{t \in T_{0}} \max _{s \in S_{t}}\left\|x_{n_{s}}\right\|_{l_{t}}+\sum_{s=1, T_{s} \neq \emptyset}^{m}\left\|x_{n_{s}}\right\|_{l_{t_{s}}}+2\right] \\
\leq & \frac{\left|z_{t_{0}}^{*}(y)\right|}{\sqrt{f(k)}}+\frac{\max _{s \leq m}\left|a_{s}\right|}{\sqrt{f(k)}} \min (m, k) \max _{s \leq m, t_{0}<t \leq k}\left\|x_{s}\right\|_{l_{t}}+2 \max _{s \leq m}\left|a_{s}\right|,
\end{aligned}
$$

which proves our claim (b).

Lemma 4.7. Assume that $\left(x_{n}\right)$ is an SRIS of constant $c, c>0$ and assume $z^{*} \in \Gamma^{*}$. As before write $z^{*}$ as

$$
z^{*}=\frac{1}{\sqrt{f(k)}} \sum_{t=1}^{k} z_{t}^{*} \in \Gamma_{k}^{*}
$$

with $z_{1}^{*} \in A_{l_{1}}^{*}$, and $l_{1}=j_{2 k^{\prime}}$, for some $k^{\prime} \geq k$, and $z_{i}^{*} \in A_{l_{i}}^{*}$, with $l_{i}=\sigma\left(z_{1}^{*}, z_{2}^{*}, \ldots, z_{i-1}^{*}\right)$, for $i=2, \ldots k$ and assume that

$$
t_{0}=\min \left\{t=1, \ldots k: z_{t}^{*}(y) \neq 0\right\},
$$

exists (otherwise $z^{*}(y)=0$ ). Let $m$ and $m \leq n_{1}<n_{2}<\ldots n_{m}$ be in $\mathbb{N},\left(a_{s}\right)_{s=1}^{m} \subset \mathbb{R}$ and assume that the numbers $j_{\tilde{p}\left(n_{s}\right)}$ (as chosen in Definition 4.4) are all different from the numbers $l_{t_{0}+1}, l_{t_{0}+2}, \ldots . l_{k}$.

Then it follows that

$$
\begin{aligned}
\left|z_{t}^{*}\left(x_{n_{s}}\right)\right| & \leq \varepsilon_{n_{1}}, \text { for } t=t_{0}+1, t_{0}+2, \ldots k \text { and } s=1,2 \ldots m, \text { and } \\
\left|z^{*}(y)\right| & \leq \frac{\left|z_{t_{0}}^{*}(y)\right|}{\sqrt{f(k)}}+3 \max _{s \leq m}\left|a_{s}\right|, \text { where } y=\sum_{s=1}^{m} a_{s} x_{n_{s}} .
\end{aligned}
$$


Remark. The assumption of Lemma 4.7 are for example satisfied if in Definition 4.4 the numbers $\tilde{p}(n), n \in \mathbb{N}$, are chosen to be odd numbers (since the image of $\sigma$ is a subset of $\left.\left\{j_{2 i}: i \in \mathbb{N}\right\}\right)$.

Proof of Lemma 4.7. We need to estimate $\left\|x_{n_{s}}\right\|_{l_{t}}$ for $s \in\{1,2 \ldots\}$ and $t=t_{0}+1, t_{0}+$ $2, \ldots k$ and then apply (55). Recall that

$$
x_{n_{s}}=\frac{\tilde{g}\left(\tilde{m}\left(n_{s}\right)\right)}{\tilde{m}\left(n_{s}\right)} \sum_{r=1}^{\tilde{m}\left(n_{s}\right)} \tilde{x}_{\tilde{s}_{r}\left(n_{s}\right)}
$$

where $\tilde{g}:[1, \infty) \rightarrow[1, \infty)$ is an increasing function with $\tilde{g}(\xi) \leq f(\xi), \xi \geq 1$ and so that $k_{n_{s}}=j_{\tilde{p}\left(n_{s}\right)},\left(\tilde{x}_{n}\right)$ is an RIS of constant $c$, and $\tilde{m} \in\left[j_{\tilde{p}\left(n_{s}\right)}, N\left(j_{\tilde{p}\left(n_{s}\right)}, \varepsilon_{\tilde{p}\left(n_{s}\right)}\right)\right]$, and $\tilde{m}\left(n_{s}\right) \leq \tilde{s}_{1}\left(n_{s}\right)<\ldots<\tilde{s}_{\tilde{m}}\left(n_{s}\right)$.

We note that either $l_{t}<k_{n_{s}}=j_{\tilde{p}\left(n_{s}\right)}$, then, since $z_{s}$ is an $\ell_{1}^{j_{\tilde{p}\left(n_{s}\right)}}$-average, we deduce from Lemma 4.2, and (401) that

$$
\left\|x_{n_{s}}\right\|_{l_{t}} \leq \frac{2}{f\left(l_{t}\right)} \leq \frac{2}{f\left(\sigma\left(z_{1}^{*}, z_{2}^{*}, \ldots z_{t-1}^{*}\right)\right)} \leq \varepsilon_{n_{1}} .
$$

Or we have that $l_{t}>k_{n_{s}}=j_{\tilde{p}\left(n_{s}\right)}$. This implies by (39) that

$$
f\left(l_{t}\right) \geq f\left(j_{\tilde{p}\left(n_{s}\right)+1}\right) \geq \frac{2}{\varepsilon_{\tilde{p}\left(n_{s}\right)+1}} N\left(j_{\tilde{p}\left(n_{s}\right)}, \varepsilon_{\tilde{p}\left(n_{s}\right)}\right) \geq \frac{2 \tilde{m}\left(n_{s}\right)}{\varepsilon_{\tilde{p}\left(n_{s}\right)+1}} \geq \frac{2 \tilde{m}\left(n_{s}\right)}{\varepsilon_{n_{1}}} .
$$

But then it follows from (65), Lemma 4.5 (b), and (45) that

$$
\left\|x_{n_{s}}\right\|_{l_{t}} \leq 2 \frac{\tilde{g}\left(\tilde{m}\left(n_{s}\right)\right)}{\tilde{m}\left(n_{s}\right)} \leq 2 \frac{f\left(\tilde{m}\left(n_{s}\right)\right)}{\tilde{m}\left(n_{s}\right)} \leq 2 \frac{f\left(k_{n_{s}}\right)}{k_{n_{s}}} \leq \varepsilon_{n_{1}} .
$$

Thus, (55) yields

$$
\left|\tilde{z}^{*}\left(x_{n_{s}}\right)\right| \leq \frac{\left|z_{t_{0}}^{*}(y)\right|}{\sqrt{f(k)}}+\frac{\max _{s \leq m}\left|a_{s}\right|}{\sqrt{f(k)}} \min (m, k) \varepsilon_{n_{1}}+2 \max _{s \leq m}\left|a_{s}\right| \leq \frac{\left|z_{t_{0}}^{*}(y)\right|}{\sqrt{f(k)}}+3 \max _{s \leq m}\left|a_{s}\right|,
$$

which proves our claim.

We now can formulate and prove our Key Lemma.

Lemma 4.8. For each $c \in(0,1]$ there is a constant $C=C_{c}>0$ so that the following holds.

Let $\left(x_{n}\right)$ be a c-SRIS, and assume that the $\tilde{p}(n), n \in \mathbb{N}$, as in Definition 4.4 are chosen to be odd numbers. Let $m \leq n_{1}<n_{2}<\ldots n_{m}$ be in $\mathbb{N}$ and put $y=\sum_{s=1}^{m} x_{n_{s}}$. Then

a) $c \frac{m}{f(m)} \leq\|y\| \leq C \frac{m}{f(m)}$, if $c<1$ and $\left(1-\varepsilon_{n_{1}}\right) \frac{m}{f(m)} \leq\|y\| \leq C \frac{m}{f(m)}$, if $c=1$.

b) For $l \in \mathbb{N}, l \geq 2$

$$
\|y\|_{l} \leq \begin{cases}\frac{C}{f(l)} \frac{m}{f(m / \min (2 l, m))} & \text { if } f(l) \leq m / \varepsilon_{n_{1}}, \\ 2 C & \text { if } f(l) \geq m / \varepsilon_{n_{1}} .\end{cases}
$$


Remark. Of course we can (and will later) replace $2 C$ in the second case of (b) in Lemma 4.8 by an another constant. Nevertheless the " $2 C$ " is needed so that the induction argument in the proof will work out.

Proof. The first inequality in (a) follows from the fact that

$$
\|y\| \geq\|y\|_{m} \geq \frac{1}{f(m)} \sum_{i=1}^{m}\left\|x_{n_{s}}\right\| \geq c \frac{m}{f(m)}
$$

if $c<1$. A similar argument works for $c=1$.

Using the first condition in (33) it is easy to see that one can choose $m_{0} \in \mathbb{N}$ so that

$$
\begin{aligned}
f(m) \leq \frac{f(l) f(m / 2 l)}{1+\varepsilon_{0}}, 2 m \varepsilon_{m}<c \varepsilon_{m-1} \text { and } \frac{f(m)}{f(m / 4)} \leq 2, \\
\text { whenever } m \geq m_{0} \text { and } 2 \leq l \leq m / 4
\end{aligned}
$$

(note that the second inequality is satisfied as long as $c \geq 1 / m$, by the third condition in (33)). Put $C=4 m_{0}$.

We will prove the second inequality in (a) and (b) by induction for each $m \in \mathbb{N}$. If $m \leq m_{0}$ (a) and (b) are trivial.

So assume (a) and (b) are true for all $m^{\prime}<m$, for some $m \geq m_{0}$. Let $m \leq n_{1}<$ $n_{2}<\ldots<n_{m}$ be in $\mathbb{N}$ and put

$$
y=\sum_{s=1}^{m} x_{n_{s}}
$$

For $l \in \mathbb{N}, l \geq 2$, we first estimate $\|y\|_{l}$.

If $f(l) \geq m / \varepsilon_{n_{1}}$ then Lemma 4.5 (b) implies that $\|y\|_{l} \leq 2 \leq C$.

If $f(l) \leq m / \varepsilon_{n_{1}}$ it follows from the second part of (168) and Lemma 4.5 (a) that there are natural numbers $0=s_{0}<s_{1}<\ldots s_{l^{\prime}}=m$, with $l^{\prime}=\min (2 l, m)$ so that

$$
\|y\|_{l} \leq \frac{\varepsilon_{n_{1}-1}}{f(l)}+\frac{1}{f(l)} \sum_{j=1}^{l^{\prime}}\left\|\sum_{s=s_{j-1}+1}^{s_{j}} x_{n_{s}}\right\| .
$$

If $l \geq m / 4$ then by the third part of (68)

$$
\|y\|_{l} \leq \frac{\varepsilon_{n_{1}-1}}{f(l)}+\frac{m}{f(l)} \leq \frac{\varepsilon_{n_{1}-1}}{f(l)}+\frac{m}{f(m / 4)} \leq \frac{\varepsilon_{n_{1}-1}}{f(l)}+2 \frac{m}{f(m)} \leq C \frac{m}{f(m)}
$$


If $l \leq m / 4$ we are using the induction hypothesis and the fact that the map $[1, \infty) \ni$ $x \mapsto x / f(x)$ is concave to obtain

$$
\begin{aligned}
\|y\|_{l} & \leq \frac{\varepsilon_{n_{1}-1}}{f(l)}+\frac{1}{f(l)} \sum_{j=1}^{l^{\prime}}\left\|\sum_{s=s_{j-1}+1}^{s_{j}} x_{n_{s}}\right\| \\
& \leq \frac{\varepsilon_{n_{1}-1}}{f(l)}+\frac{C}{f(l)} \sum_{j=1}^{l^{\prime}} \frac{s_{j}-s_{j-1}}{f\left(s_{j}-s_{j-1}\right)} \\
& \leq \frac{\varepsilon_{n_{1}-1}}{f(l)}+\frac{C}{f(l)} l^{\prime} \frac{m / l^{\prime}}{f\left(m / l^{\prime}\right)} \\
& \left.\leq \frac{\varepsilon_{0}}{f(2)}+\frac{C}{1+\varepsilon_{0}} \frac{m}{f(m)} \text { by first condition in (68) }\right) \\
& \leq C \frac{m}{f(m)},
\end{aligned}
$$

for the last inequality note that

$$
\frac{\varepsilon_{0}}{f(2)} \leq C \frac{\varepsilon_{0}}{1+\varepsilon_{0}} \frac{2}{f(2)}=C\left[1-\frac{1}{1+\varepsilon_{0}}\right] \frac{2}{f(2)} \leq C\left[1-\frac{1}{1+\varepsilon_{0}}\right] \frac{m}{f(m)} .
$$

This proves that $\|y\|_{l} \leq C m / f(m)$, for every $l \geq 2$. Together with Lemma 4.7 (which estimates $\|y\|_{\Gamma_{k}^{*}}$ for $k \in \mathbb{N}$ ) this yields that $\|y\| \leq C m / f(m)$. That finishes the induction step and the proof of (a).

Part (b) follows if $f(l) \geq m / \varepsilon_{n_{1}}$ directly from Lemma 4.5 (b). If $f(l) \leq m / \varepsilon_{n_{1}}$ we apply Lemma 4.5 (a), the concavity of the map $[1, \infty) \ni x \mapsto x / f(x)$ and part (a) of this lemma, to obtain for some choice of natural numbers $0=s_{0}<s_{1}<\ldots s_{l^{\prime}}=m$, with $l^{\prime}=\min (2 l, m)$ so that

$$
\begin{aligned}
\|y\|_{l} & \leq \frac{\varepsilon_{n_{1}-1}}{f(l)}+\frac{1}{f(l)} \sum_{j=1}^{l^{\prime}}\left\|\sum_{s=s_{j-1}+1}^{s_{j}} x_{n_{s}}\right\| \\
& \leq \frac{\varepsilon_{n_{1}-1}}{f(l)}+\frac{C}{f(l)} \sum_{j=1}^{l^{\prime}} \frac{s_{j}-s_{j-1}}{f\left(s_{j}-s_{j-1}\right)} \\
& \leq \frac{\varepsilon_{n_{1}-1}}{f(l)}+\frac{C}{f(l)} \frac{m}{f\left(m / l^{\prime}\right)} \leq \frac{2 C}{f(l)} \frac{m}{f\left(m / l^{\prime}\right)},
\end{aligned}
$$

which proves our claim.

Remark. Following now the proof in [GM] (from Lemma 7 in [GM] on) one deduces that $\mathcal{G M}$, as defined here has also no unconditional basis sequence. In Section [6 (see Theorem 6.1) we will prove that in every block subspace of $\mathcal{G M}$ there are two seminormalized block sequence $\left(u_{n}\right)$ and $\left(v_{n}\right)$, which are intertwinned, i.e. $u_{1}<v_{1}<$ 
$u_{2}<v_{2}<\ldots$, with the property that for some constants $0<c, C<\infty$

$$
\left\|\sum_{s=1}^{l} u_{n_{s}}+v_{n_{s}}\right\| \geq c \frac{l}{\sqrt{f(l)}} \text { and }\left\|\sum_{s=1}^{l} u_{n_{s}}-v_{n_{s}}\right\| \leq C \frac{l}{f(l)} .
$$

for all $l \in \mathbb{N}$ and all choices of $l \leq n_{1}<n_{2}<\ldots n_{l}$ in $\mathbb{N}$. This certainly implies that $\mathcal{G M}$ has no unconditional block sequence.

We do not know whether or not $\mathcal{G} \mathcal{M}$ is HI, but we suspect it is. The point is that to use spreading models and other refinements, we needed to pass to subsequences of the Rapidly Increasing Sequences as defined in GM. Therefore we lost the freedom to pick the vectors of an RIS-sequence in arbitrary subspaces, as would be needed to repeat Gowers-Maurey's proof that $G M$ is HI.

Nevertheless, Gowers' first dichotomy yields that $\mathcal{G} \mathcal{M}$ contains at least an infinite dimensional block subspace which is HI.

\section{Yardstick VeCtors in $\mathcal{G} \mathcal{M}$}

We will prove that every block basis in $\mathcal{G M}$ has a further block basis whose spreading model is equivalent to the unit vector basis of $S$. Thus, we can define in $\mathcal{G M}$ the yardsticks as introduced in Section 2, The following observation follows from Lemmas 4.5 and 4.8, and an argument in [AS2.

Proposition 5.1. Assume that $\left(x_{n}\right)$ is a c-RIS in $\mathcal{G M}$, for which the following condition is satisfied:

There exists a constant $C^{\prime} \geq 1$, so that for all $m, k \in \mathbb{N}, m \leq n_{1}<n_{2}<\ldots n_{m}$ in $\mathbb{N}$, all $\left(a_{i}\right)_{i=1}^{m}$ and all $z^{*} \in \Gamma_{k}^{*}$, it follows that

$$
\left|z^{*}\left(\sum_{s=1}^{m} a_{s} x_{n_{s}}\right)\right| \leq \frac{1}{\sqrt{f(k)}} \max _{j \in J}\left\|\sum_{s=1}^{m} a_{s} x_{n_{s}}\right\|_{j}+C^{\prime} \max _{s \leq m}\left|a_{s}\right| .
$$

Then the spreading model of $\left(x_{n}\right)$ is equivalent to the unit vector basis of $S$. More precisely there is a constant $C$ so that for every c-SRIS $\left(x_{n}\right)$ in $\mathcal{G M}$

$$
c^{\prime}\left\|\sum_{s=1}^{m} a_{s} e_{s}\right\|_{S} \leq\left\|\sum_{s=1}^{m} a_{s} x_{n_{s}}\right\|_{\mathcal{G M}} \leq C\left\|\sum_{s=1}^{m} a_{s} e_{s}\right\|_{S},
$$

whenever $m \leq n_{1}<n_{2}<\ldots n_{m}$ are in $\mathbb{N}$ and $\left(a_{s}\right)_{s=1}^{m} \subset \mathbb{R}$, where $c^{\prime}=c$ if $c<1$ and $c^{\prime}=1-\varepsilon_{n_{1}}$ if $c=1$.

Remark. Note that Lemma 4.7 and the remark thereafter establishes a case in which the assumption (69) is satisfied.

Proof of Proposition 5.1. Consider the norm $\langle\cdot\rangle$ on $c_{00}$ given by the implicit equation

$$
\left\langle\langle x\rangle=\max \left(\|x\|_{\infty}, \max _{\substack{l \in \mathbb{N}, l \geq 3 \\ E_{1}<E_{2}<\ldots E_{l}}} \frac{1}{f(l / 2)} \sum_{j=1}^{l}\left\langle\left\langle E_{i}(x)\right\rangle\right), x \in c_{00}\right.\right.
$$


and recall [AS2, Lemma 3.3] which states that $\langle\langle\cdot\rangle$ is an equivalent norm on $S$.

We put

$$
C^{\prime \prime}=C^{\prime} \frac{\sqrt{f(2)}}{\sqrt{f(2)}-1}
$$

By induction we will show for each $m \in \mathbb{N}$ and all choices of $\left(a_{s}\right)_{s=1}^{m} \subset \mathbb{R}$ and $m \leq n_{1}<n_{2}<\ldots n_{m}$ in $\mathbb{N}$, that

$$
c^{\prime}\left\|\sum_{s=1}^{m} a_{s} e_{s}\right\|_{S} \leq\left\|\sum_{s=1}^{m} a_{s} x_{n_{s}}\right\|_{\mathcal{G M}} \leq C^{\prime \prime}\left\langle\left\langle\sum_{s=1}^{m} a_{s} e_{s}\right\rangle\right\rangle\left(1+\frac{2}{c} \sum_{s=1}^{m} \varepsilon_{n_{s}}\right) .
$$

This will, together with the above cited result from AS2, prove our claim. The first inequality in (71) is clear, and it is also clear that (71) holds for $m=1$. So assume that (71) holds for all $m^{\prime}<m, m \geq 2, m^{\prime} \leq n_{1}<n_{2}<\ldots n_{m^{\prime}}$ in $\mathbb{N}$, and $\left(a_{s}\right)_{s=1}^{m^{\prime}} \subset \mathbb{R}$. Let $m \leq n_{1}<n_{2}<\ldots n_{m},\left(a_{s}\right)_{s=1}^{m} \subset \mathbb{R}$ and put $y=\sum_{s=1}^{m} a_{s} x_{n_{s}}$. We distinguish between two cases: If

$$
C^{\prime \prime} \max _{s \leq m}\left|a_{s}\right| \geq \max _{l \in \mathbb{N}, l \geq 2}\|y\|_{l}
$$

then we note that for all $l \in \mathbb{N}, l \geq 2$,

$$
\|y\|_{l} \leq C^{\prime \prime} \max \left|a_{s}\right| \leq C^{\prime \prime}\left\langle\left\langle\sum_{s=1}^{m} a_{s} e_{s}\right\rangle\right\rangle
$$

and, thus, for any $k \in \mathbb{N}, k \geq 2$, and $z^{*} \in \Gamma_{k}^{*}$, it follows from our assumption (69) that

$$
\left|z^{*}(y)\right| \leq \frac{1}{\sqrt{f(2)}} \max _{j \in J}\|y\|_{j}+C^{\prime} \max _{s \leq m}\left|a_{s}\right| \leq\left[\frac{C^{\prime \prime}}{\sqrt{f(2)}}+C^{\prime}\right] \max _{s \leq m}\left|a_{s}\right|=C^{\prime \prime} \max _{s \leq m}\left|a_{s}\right|
$$

If

$$
C^{\prime \prime} \max _{s \leq m}\left|a_{s}\right|<\max _{l \in \mathbb{N}, l \geq 2}\|y\|_{l}
$$

we proceed as follows.

If $l \in \mathbb{N}$, with $f(l) \geq 2 m / \varepsilon_{n_{1}}$, then Lemma 4.5 (b) implies that

$$
\|y\|_{l} \leq 2 \max _{i \leq m}\left|a_{s}\right| \leq 2\left\langle\left\langle\sum_{i=1}^{m} a_{i} e_{i}\right\rangle\right\rangle \leq C^{\prime \prime}\left\langle\left\langle\sum_{i=1}^{m} a_{i} e_{i}\right\rangle\right\rangle
$$


If $l \geq 2$ and $f(l) \leq 2 m / \varepsilon_{n_{1}}$, then Lemma 4.5 (a) yields for some choice of $0=s_{0}<$ $s_{1}<s_{2}<\ldots s_{l^{\prime}}$ with $l^{\prime}=\min (m, 2 l)$, that

$$
\begin{aligned}
\|y\|_{l} & \leq \frac{1}{f(l)}\left[\sum_{t=1}^{l^{\prime}}\left\|\sum_{s=s_{t-1}+1}^{s_{t}} a_{s} x_{n_{s}}\right\|+\frac{2}{c} \sum_{t \leq l^{\prime}, s_{t}=1+s_{t-1}} \varepsilon_{n_{s_{t}}}\left|a_{s_{t}}\right|\right] \\
& \left.\left.\leq \frac{C^{\prime \prime}}{f(l)}\left[\sum_{t=1}^{l^{\prime}} \| \sum_{s=s_{t-1}+1}^{s_{t}} a_{s} e_{s}\right\rangle\right\rangle\left(1+\frac{2}{c} \sum_{s=1}^{m} \varepsilon_{n_{s}}\right)\right]
\end{aligned}
$$

(By applying the induction hypothesis in cases that $s_{t} \geq 2+s_{t-1}$ )

$$
\leq C^{\prime \prime}\left\langle\left\langle\sum_{s=1}^{m} a_{s} e_{s}\right\rangle\right\rangle\left(1+\frac{2}{c} \sum_{s=1}^{m} \varepsilon_{n_{s}}\right) \text {. }
$$

Our assumption (69) yields for $k \in \mathbb{N}, k \geq 2$ and $z^{*} \in \Gamma_{k}^{*}$, that

$$
\left|z^{*}(y)\right| \leq \frac{1}{\sqrt{f(k)}} \max _{j \in \mathbb{N}}\|y\|_{j}+C^{\prime} \max _{s \leq m}\left|a_{s}\right| \leq\left[\frac{1}{\sqrt{f(2)}}+\frac{C^{\prime}}{C^{\prime \prime}}\right] \max _{j \in \mathbb{N}}\|y\|_{j}=\max _{j \in \mathbb{N}}\|y\|_{j},
$$

which together with (72), finishes the proof of our induction step.

Lemma 3.1 and Proposition 5.1 imply therefore

Corollary 5.2. There is a constant D, so that for every asymptotically isometric SRIS $\bar{x}=\left(x_{n}\right)$ in $\mathcal{G M}$, for which $\tilde{p}(n)$ is odd for all $n \in \mathbb{N}$, and every $l \in \mathbb{N}$ and any $s_{1}<s_{2}<\ldots s_{l}$ in $\mathbb{N}$ we have

$$
\frac{1}{2} \leq\left\|y_{\bar{x}^{\prime}}\left(j_{s_{1}}, j_{s_{2}}, \ldots j_{s_{l}}\right)\right\|_{j_{s_{i}}} \leq\left\|y_{\bar{x}^{\prime}}\left(j_{s_{1}}, j_{s_{2}}, \ldots j_{s_{l}}\right)\right\| \leq \frac{D}{2}, \text { for all } i=1,2, \ldots l
$$

where $\bar{x}^{\prime}$ is a far enough out starting tail subsequence of $\bar{x}$.

\section{Construction of two Equivalent intertwined Sequences}

We now want to construct in a given block subspace $Y$ of $\mathcal{G} \mathcal{M}$ two seminormalized block sequences $\left(u_{n}\right)$ and $\left(v_{n}\right)$, which are equivalent and so that $u_{1}<v_{1}<u_{2}<\ldots$

Let $\bar{x}=\left(x_{i}\right)$ be any asymptotically isometric SRIS in $Y$, so that $\tilde{p}(n)$ is odd for $n \in \mathbb{N}$. Using Proposition 5.1 and the remark thereafter, it follows that the spreading model of $\bar{x}$ is equivalent to the unit vector basis of $S$, and, since Corollary 5.2 applies we let $D<\infty$ be chosen so that (73) holds true.

By induction we choose a block sequence $\left(z_{n}\right)$ of $\bar{x}$. The vectors $u_{n}$ and $v_{n}$ will then be chosen so that $u_{n}<v_{n}$ and $z_{n}=u_{n}+v_{n}$.

For $n=1$ we first choose $k_{1}^{\prime}$, so that $f\left(k_{1}^{\prime}\right) / k_{1}^{\prime}<\varepsilon_{1}^{2}$ (which means that $k_{1}^{\prime}$ satisfies condition (45) for $n=1$ ), and then let

$$
z_{1}=\frac{1}{D} y_{\bar{x}^{(1)}}\left(j_{2 q_{1}(1)}\right)=\frac{1}{D} \frac{f\left(j_{2 q_{1}(1)}\right)}{j_{2 q_{1}(1)}} \sum_{t=1}^{j_{2 q_{1}(1)}} \bar{x}_{t}^{(1)}
$$

where $q_{1}(1) \in \mathbb{N}$ is chosen large enough so that $y\left(j_{2 q_{1}(1)}\right)$ is an $\ell_{1}^{k_{1}}$-average of constant $1-\varepsilon_{1}$, with $k_{1} \geq k_{1}^{\prime}$ (using Lemma 2.9), $\bar{x}^{(1)}$ is a tail subsequence of $\bar{x}$, which starts 
far enough out so that $\left\|z_{1}\right\| \leq 1$ and so that $z_{1}$ is an $\ell_{1}^{k_{1}}$-average of constant $\frac{1}{D}$ (using Proposition 5.1 and Corollary 5.2). Finally we choose

$$
u_{1}=\frac{1}{D} \frac{f\left(j_{2 q_{1}(1)}\right)}{j_{2 q_{1}(1)}} \sum_{t=1}^{j_{2 q_{1}(1)} / 2} \bar{x}_{t}^{(1)} \text { and } v_{1}=\frac{1}{D} \frac{f\left(j_{2 q_{1}(1)}\right)}{j_{2 q_{1}(1)}} \sum_{t=1+\left(j_{2 q_{1}(1)} / 2\right)}^{j_{2 q_{1}(1)}} \bar{x}_{t}^{(1)}
$$

(recall that the elements of $J$ are even).

Assume now that for some $n \geq 2$, we have chosen $z_{1}<z_{2}<\ldots z_{n-1}$ in $B_{\mathcal{G M}}$, and assume that the following conditions are satisfied:

- for each $i<n, z_{i}$ is an $\ell_{1}^{k_{i}}$-average of constant $1 / D$, so that

$$
f\left(k_{i}\right) / k_{i}<\varepsilon_{i}^{2} \text { and } f\left(\varepsilon_{i} \sqrt{k_{i}}\right)>\frac{1}{\varepsilon_{i}^{2}} \max \operatorname{supp}\left(z_{i-1}\right) \text {, if } i \geq 2
$$

(in other words $z_{1}<z_{2}<\ldots z_{n-1}$ satisfies the condition (45) of the first $n-1$ elements of an RIS).

- secondly $z_{i}$ is of the form

$$
z_{i}=u_{i}+v_{i}=\frac{1}{D} y_{\bar{x}^{(i)}}\left(j_{2 q_{i}(1)}, j_{2 q_{i}(2)}, \ldots j_{2 q_{i}\left(l_{i}\right)}\right)
$$

where $l_{i}$, and $q_{i}(1)<q_{i}(2)<\ldots<q_{i}\left(l_{i}\right)$ are in $\mathbb{N}$ and $\bar{x}^{(i)}$ is a tail subsequence of $\bar{x}$, starting far enough out to ensure that $\left(z_{i}\right)_{i=1}^{n-1}$ is a block sequence and is in $B_{\mathcal{G M}}$ (using Corollary 5.2). By Definition 2.7 of the yardstick vectors in Section 2 we can write $z_{i}$ as $z_{i}=\sum_{r=1}^{l_{i}} z(i, r)$ where the $z(i, r), r=1,2 \ldots l_{i}$, have pairwise disjoint support and so that for each $r \leq l_{i}$ the vector $z(i, r)$ is of the form

$$
z(i, r)=\frac{1}{D} \frac{f\left(j_{2 q_{i}\left(r_{t}\right)}\right)}{j_{2 q_{i}\left(r_{t}\right)}} \sum_{s=1}^{j_{2 q_{i}(r)}} x(i, r, s)
$$

where $x(i, r, q), q=1,2, \ldots j_{2 q_{i}(r)}$ are elements of the sequence $\bar{x}^{(i)}$, and we have

$$
\begin{aligned}
& u_{i}=\frac{1}{D} \sum_{r=1}^{l_{i}} \frac{f\left(j_{2 q_{i}(r)}\right)}{j_{2 q_{i}(r)}} \sum_{s=1}^{j_{2 q_{i}(r)} / 2} x(i, r, s) \text { and } \\
& v_{i}=\frac{1}{D} \sum_{r=1}^{l_{i}} \frac{f\left(j_{2 q_{i}(r)}\right)}{j_{2 q_{i}(r)}} \sum_{s=1+\left(j_{2 q_{i}(r)} / 2\right)}^{j_{2 q_{i}(r)}} x(i, r, s) .
\end{aligned}
$$

- moreover we assume that so far the following condition is satisfied:

For each sequence $\bar{\iota}=\left(i_{t}: t=1,2 \ldots, l\right) \subset\{1,2, \ldots, n-1\}$, with $1 \leq i_{1}<i_{2}<$ $\ldots i_{l} \leq n-1$, and for each $\bar{\rho}=\left(\rho_{t}: t=1,2 \ldots, l\right) \in\{-1,1\}^{l}$ there is a sequence of functionals $\bar{z}^{*}(\bar{l}, \bar{\rho})=\left(z_{t}^{*}: t=1,2, \ldots l\right)=\left(z_{(\bar{\imath}, \bar{\rho})}^{*}(t): t=1,2 \ldots, l\right)$ in $B_{\mathcal{G M}^{*}}$ so that 
for all $t=1,2 \ldots, l-1$ :

(a) $\operatorname{supp}\left(z_{t}^{*}\right) \subset \bigcup_{s=1}^{\left.j_{2 q_{i}\left(r_{t}\right)}\right)} \operatorname{ran}\left(x\left(i_{t}, r_{t}, s\right)\right)$, for some $r_{t} \in\left\{1,2 \ldots l_{i_{t}}\right\}$,

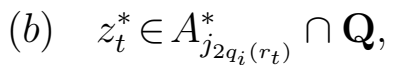

(c) $z_{t}^{*}\left(z\left(i_{t}, r_{t}\right)\right) \rho_{t} \geq \frac{1}{2 D}, z_{t}^{*}\left(u\left(i_{t}\right)\right)=z_{t}^{*}\left(v\left(i_{t}\right)\right)=\frac{1}{2} z_{t}^{*}\left(z\left(i_{t}, r_{t}\right)\right)$, and,

(d) if $t \geq 2$, then $2 j_{2 q_{i}\left(r_{t}\right)}=\sigma\left(\rho_{1} z_{1}^{*}, \rho_{2} z_{2}^{*}, \ldots, \rho_{t-1} z_{t-1}^{*}\right)$.

In order to choose $z_{n}$ we proceed as follows. We first choose $k_{n}^{\prime} \in J$ so that $f\left(k_{n}^{\prime}\right) / k_{n}^{\prime}<\varepsilon_{n}^{2}$ and $f\left(\varepsilon_{n} \sqrt{k_{n}^{\prime}}\right) \geq \frac{1}{\varepsilon_{n}^{2}} \max \operatorname{supp}\left(z_{n-1}\right)$. Assume that $q_{n} \in \mathbb{N}$ satisfies the following properties:

$$
j_{q_{n}} \geq k_{n}^{\prime} \text { and } \sqrt{f\left(j_{q_{n}} \varepsilon_{n}\right)}>\frac{\max \operatorname{supp}\left(z_{n-1}\right)}{\varepsilon_{n}} .
$$

For each increasing sequence $\bar{\imath}=\left(i_{t}: t=1,2 \ldots, l\right) \subset\{1,2, \ldots n-1\}$, and each $\bar{\rho}=\left(\rho_{t}: t=1,2 \ldots, l\right) \subset\{ \pm 1\}$ we can assume that

$$
\sigma\left(z_{(\bar{l}, \bar{\rho})}^{*}(1), z_{(\bar{l}, \bar{\rho})}^{*}(2), \ldots, z_{(\bar{\nu}, \bar{\rho})}^{*}(l)\right) \geq j_{q_{n}+1},
$$

for any $\bar{\imath}=\left(i_{t}: t=1,2 \ldots, l\right) \subset\{1,2, \ldots n-1\}$, with $1 \leq i_{1}<i_{2}<\ldots i_{l} \leq n-1$, and for each $\bar{\rho}=\left(\rho_{t}: t=1,2 \ldots, l\right) \subset\{ \pm 1\}$. Note that this can be accomplished by only perturbing the last element $z_{(\bar{\imath}, \bar{\rho})}^{*}(l)$, and thus still satifying condition (179) (c) (and all the other conditions of (79) ). Then we consider the set

$$
\Sigma_{n}=\left\{\sigma\left(z_{(\bar{\iota}, \bar{\rho})}^{*}(1), z_{(\bar{\iota}, \bar{\rho})}^{*}(2), \ldots, z_{(\bar{\imath}, \bar{\rho})}^{*}(l)\right): \begin{array}{c}
\bar{\iota}= \\
\left(i_{t}: t \leq l\right) \subset\{1, \ldots n-1\} \text { increasing } \\
\bar{\rho}=\left(\rho_{t}: t=1,2 \ldots, l\right) \subset\{ \pm 1\}
\end{array}\right\}
$$

and order it into

$$
j_{2 q_{n}(1)}<j_{2 q_{n}(2)}<\ldots<j_{2 q_{n}\left(l_{n}\right)} .
$$

We then choose a tail subsequence $\bar{x}^{(n)}$ of $\bar{x}$ whose first element starts after $z_{n-1}$ and so that its first $\sum_{r=1}^{l_{n}} j_{2 q_{n}(r)}$ elements are $\left(1+\varepsilon_{n}\right) C(C$ as in Proposition 5.1) equivalent to the first $\sum_{r=1}^{l_{n}} j_{2 q_{n}(2)}$ elements of $S$, and then put

$$
z_{n}=\frac{1}{D} y_{\bar{x}}\left(j_{2 q_{n}(1)}, j_{2 q_{n}(2)}, \ldots, j_{2 q_{n}\left(l_{n}\right)}\right) .
$$

Then $z_{n}>z_{n-1}$ and $\left\|z_{n}\right\|_{\mathcal{G M}} \leq 1$ by Proposition 5.1. Lemma 2.9, Proposition 5.1 and Corollary 5.2 yield that $z_{n}$ is an $\ell_{1}^{k_{n}}$ average of constant $\frac{1}{D}$, with $k_{n} \geq k_{n}^{\prime}$.

As before we can, by the definition of the yardstick vectors, write $z_{n}$ as

$$
z_{n}=\sum_{r=1}^{l_{n}} z(n, r)
$$

where the $z(n, r)$ have pairwise disjoint support and $z(n, r)$ is for each $r \leq l_{i}$ of the form

$$
z(i, r)=\frac{1}{D} \frac{f\left(j_{2 q_{n}\left(r_{t}\right)}\right)}{j_{2 q_{n}\left(r_{t}\right)}} \sum_{s=1}^{j_{2 q_{n}(r)}} x(i, n, s)
$$


where $\left.x(i, n, q), q=1,2, \ldots j_{2 q_{n}(r)}\right)$ are elements of the sequence $\bar{x}^{(n)}$, and we let

$$
\begin{aligned}
& u_{n}=\frac{1}{D} \sum_{r=1}^{l_{n}} \frac{f\left(j_{2 q_{n}(r)}\right)}{j_{2 q_{n}(r)}} \sum_{s=1}^{j_{2 q_{n}(r)} / 2} x(n, r, s) \text { and } \\
& v_{n}=\frac{1}{D} \sum_{r=1}^{l_{n}} \frac{f\left(j_{2 q_{n}(r)}\right)}{j_{2 q_{n}(r)}} \sum_{t=s+\left(j_{2 q_{n}(r)} / 2\right)}^{j_{2 q_{n}(r)}} x(n, r, s) .
\end{aligned}
$$

It is now easy to find for every $\bar{\imath}=\left(i_{t}: t=1,2 \ldots, l\right) \subset\{1,2, \ldots, n\}$, with $1 \leq$ $i_{1}<i_{2}<\ldots i_{l} \leq n$, and for each $\bar{\rho}=\left(\rho_{t}: t=1,2 \ldots, l\right) \in\{-1,1\}^{l}$ a sequence of functionals $\bar{z}^{*}(\bar{\iota}, \bar{\rho})=\left(z_{t}^{*}: t=1,2, \ldots l\right)=\left(z_{(\bar{l}, \bar{\rho})}^{*}(t): t=1,2 \ldots, l\right) \in B_{\mathcal{G} \mathcal{M}^{*}}$ so that (79) holds. Indeed, if the last element $i_{l}<n$, then we already have chosen $\bar{z}^{*}(\bar{\iota}, \bar{\rho})$. So let us assume $i_{l}=n$. Let $\bar{\iota}^{\prime}=\left(i_{t}: t=1,2 \ldots, l-1\right)$ and $\bar{\rho}^{\prime}=\left(\rho_{t}: t=1,2 \ldots, l-1\right)$. If $\bar{\iota}^{\prime}$ and $\bar{\rho}^{\prime}$ are empty we choose $r=1$. Otherwise we choose $r=r(\bar{\iota}, \bar{\rho}) \in\left\{1,2 \ldots, l_{n}\right\}$ so that

$$
j_{2 q_{n}(r)}=\sigma\left(z_{\left(\bar{\iota}^{\prime}, \bar{\rho}^{\prime}\right)}^{*}(1), z_{\left(\bar{t}^{\prime}, \bar{\rho}^{\prime}\right)}^{*}(2), \ldots, z_{\left(\bar{\iota}^{\prime}, \bar{\rho}^{\prime}\right)}^{*}(l-1)\right)
$$

(by choice of $q_{n}(i), i=1,2 \ldots l_{n}$, this is possible). Then choose for every $s=$ $1,2, \ldots, j_{2 q_{n}(r)}$, a functional $x_{s}^{*}=x^{*}(n, s, \bar{\iota}, \bar{\rho}) \in B_{\mathcal{G M}^{*}} \cap \mathbf{Q}$, so that

$$
x_{s}^{*}(x(r, n, s))=x_{s^{\prime}}^{*}\left(x\left(i, n, s^{\prime}\right)\right) \geq 1-2 \varepsilon_{n} \text {, for } s \neq s^{\prime} \text { in }\left\{1,2 \ldots j_{2 q_{n}(r)}\right\}
$$

and $\operatorname{supp}\left(x_{q}^{*}\right) \subset \operatorname{ran}(x(r, n, q))$.

Let

$$
z_{(\bar{\iota}, \bar{\rho})}^{*}(l)=\rho_{l} \frac{1}{f\left(j_{2 q_{n}(r)}\right)} \sum_{q=1}^{\left.j_{2 q_{n}(r)}\right)} x_{q}^{*}
$$

and

$$
\bar{z}_{(\bar{\imath}, \bar{\rho})}^{*}=\left(\bar{z}_{\left(\bar{\iota}^{\prime}, \bar{\rho}^{\prime}\right)}^{*}, z^{*}(\bar{\iota}, \bar{\rho})(l)\right) .
$$

It follows therefore that (179) is satisfied, which finishes our recursive definition of $z_{n}$, $u_{n}$ and $v_{n}$.

For $n \in \mathbb{N}$ and $l \in J \backslash\left\{q_{n}(1), q_{n}(2), \ldots q_{n}\left(l_{n}\right)\right\}$ we estimate $\left\|z_{n}\right\|_{l}$.

Note that the construction of the $z_{n}$ accomplishes the following:

If $m \in \mathbb{N}$ and $m \leq n_{1}<n_{2}<\ldots<n_{m}$ are in $\mathbb{N}$ and $\left(a_{s}\right)_{s=1}^{m} \subset \mathbb{R}$, we can choose $\bar{\imath}=\left(n_{s}\right)_{s 1}^{m}$ and $\bar{\rho}=\left(\operatorname{sign}\left(a_{s}\right)\right)_{s=1}^{m}$, and conclude that

$$
z^{*}=\frac{1}{\sqrt{f(k)}} \sum_{t=1}^{m} z_{(\bar{l}, \bar{\rho})}^{*}(t) \in \Gamma_{m}^{*}
$$

and

$$
\left\|\sum_{s=1}^{m} a_{s} z_{n_{s}}\right\| \geq z^{*}\left(\sum_{s=1}^{m} a_{s} z_{n_{s}}\right) \geq \frac{1}{\sqrt{f(k)}} \frac{1}{D} \sum_{s=1}^{m}\left|a_{s}\right| .
$$

After passing to a subsequence we can assume that $\left(z_{n}\right)$ has a spreading model and that it is a $\frac{1}{D}$-RIS. We define $w_{n}=u_{n}-v_{n}$. Then $\left(w_{n}\right)$ also satisfies (45) of the definition of $\frac{1}{D}$-RIS, and passing to a subsequence, we may also assume that $\left(w_{n}\right)$ has 
a spreading model satisfying (46) and is therefore also a $\frac{1}{D}$-RIS. We claim that $\left(w_{n}\right)$ satisfies the condition (69) of Proposition 5.1 and it follows therefore that $\left(w_{n}\right)$ has a spreading model which is equivalent to the unit vector basis in $S$.

We first estimate $\left\|z_{n}\right\|_{l}$ for $n \in \mathbb{N}$ and $l \in J$. Let

$$
r_{0}=\max \left\{r=1,2 \ldots l_{n}: j_{2 q_{n}(r-1)} \leq l\right\} \text { with } j_{2 q_{n}(0)}:=0 .
$$

Then by the definition of $z_{n}$ and the $z(n, r)$, by condition (38) on the sequence $\left(j_{i}\right)$, and by Lemma 2.9, it follows that $\sum_{r=r_{0}}^{l_{n}} z(n, r)$ is an $\ell_{1}^{k}$ average for some $k \geq j_{2 q_{n}\left(r_{0}\right)-1}>l$. It follows therefore from Lemma 4.2 that

$$
\left\|\sum_{r=r_{0}}^{l_{n}} z(n, r)\right\|_{l} \leq \frac{2}{f(l)}
$$

If $r=1,2 \ldots r_{0}-1$, and thus $j_{2 q_{n}(r)}<l$, we deduce from (39) that

$$
f(l) \geq f\left(j_{2 q_{n}(r)+1}\right) \geq \frac{2}{\varepsilon_{2 q_{n}(r)+1}} j_{2 q_{n}(r)}
$$

and thus, by Lemma 4.5 (b)

$$
\|z(n, r)\|_{l} \leq \frac{2 f\left(j_{2 q_{n}(r)}\right)}{j_{2 q_{n}(r)}} .
$$

It follows therefore from (87), (88) and (37) that for $l \in J \backslash\left\{q_{n}(1), q_{n}(2), \ldots q_{n}\left(l_{n}\right)\right\}$

$$
\left\|z_{n}\right\|_{l} \leq \sum_{r=1}^{l_{n}} \frac{2 f\left(j_{2 q_{n}(r)}\right)}{j_{2 q_{n}(r)}}+\frac{2}{f(l)} \leq \frac{1}{f\left(j_{2 q_{n}(1)-1}\right)}+\frac{2}{f(l)} \leq \frac{1}{j_{q_{n}}}+\frac{2}{f(l)} .
$$

Using the same argument we observe similar inequalities for $u_{n}, v_{n}, w_{n}$ :

$$
\left\|u_{n}\right\|_{l} \leq \frac{1}{j_{q_{n}}}+\frac{2}{f(l)}, \quad\left\|v_{n}\right\|_{l} \leq \frac{1}{j_{q_{n}}}+\frac{2}{f(l)} \text { and }\left\|w_{n}\right\|_{l} \leq \frac{1}{j_{q_{n}}}+\frac{2}{f(l)} .
$$

In order to verify condition (69) of Proposition 5.1 let $m \leq n_{1}<n_{2}<\ldots n_{s}$ be in $\mathbb{N}$ and $\left(a_{s}\right)_{s=1}^{m} \subset \mathbb{R} \backslash\{0\}$ we put $y=\sum_{s=1}^{m} a_{s} w_{n_{s}}$. Secondly let $k \in \mathbb{N}$ and $z^{*} \in \Gamma_{k}^{*}$. As before we write $z^{*}$ as

$$
z^{*}=\frac{1}{\sqrt{f(k)}} \sum_{t=1}^{k} z_{t}^{*} \in \Gamma_{k}^{*}
$$

with $z_{1}^{*} \in A_{l_{1}}^{*}$, and $l_{1}=j_{2 k^{\prime}}$, for some $k^{\prime} \geq k$, and $z_{i}^{*} \in A_{l_{i}}^{*}$, with $l_{i}=\sigma\left(z_{1}^{*}, z_{2}^{*}, \ldots, z_{i-1}^{*}\right)$, for $i=2, \ldots k$ and assume that

$$
t_{0}=\min \left\{t=1, \ldots k: z_{t}^{*}(y) \neq 0\right\},
$$

exists (otherwise $z^{*}(y)=0$ ). Note that the equalities in condition (79) (c) imply that $z^{*}(\bar{\iota}, \bar{\rho})(t)\left(w_{j}\right)=0$ for every $j \in \mathbb{N}$, every increasing sequence $\bar{\iota}=\left(i_{t}: t=\right.$ $1,2 \ldots, l) \subset\{1,2, \ldots$,$\} , for each \bar{\rho}=\left(\rho_{t}: t=1,2 \ldots, l\right) \in\{-1,1\}^{l}$, and for every $t=1,2 \ldots l$. So it follows that the sequence $\left(z_{1}^{*}, z_{2}^{*}, \ldots z_{t_{0}}^{*}\right)$ cannot be one of the sequences $z^{*}(\bar{\imath}, \bar{\rho})(t)$, where $\bar{\iota}=\left(i_{t}: t=1,2 \ldots, l\right) \subset\{1,2, \ldots$,$\} , is increasing and$ $\bar{\rho}=\left(\rho_{t}: t=1,2 \ldots, l\right) \in\{-1,1\}^{l}$. From the injectivity of $\sigma$ it follows therefore that the sets $\left\{l_{t}: t>t_{0}\right\}$ and the set $\left\{j_{2 q_{n}(r)}: n \in \mathbb{N}, r \leq l_{n}\right\}$ are disjoint. 
We can now apply (61) and (90) deduce that

$$
\begin{aligned}
\left|z^{*}(y)\right| \leq & \frac{\left|z_{t}^{*}(y)\right|}{\sqrt{f(k)}}+\frac{\max _{s \leq m}\left|a_{s}\right|}{\sqrt{f(k)}}\left[\sum_{t \in T_{0}} \max _{s \in S_{t}}\left\|w_{n_{s}}\right\|_{l_{t}}+1+2 m \varepsilon_{n_{1}}\right] \\
& \quad+\frac{1}{\sqrt{f(k)}} \sum_{s=s_{0}+1}^{m}\left|a_{s}\right|\left|\sum_{t \in T_{s}} z_{t}^{*}\left(w_{n_{s}}\right)\right| \\
\leq & \frac{\left|z_{t_{0}}^{*}(y)\right|}{\sqrt{f(k)}}+\frac{\max _{s \leq m}\left|a_{s}\right|}{\sqrt{f(k)}}\left[2+\sum_{t=t_{0}+1}^{k} \frac{2}{f\left(l_{k}\right)}+\sum_{s=1}^{s_{0}} \frac{1}{j_{q_{n_{s}}}}+\sum_{s=s_{0}+1}^{m} \frac{k}{j_{q_{s}}}\right]
\end{aligned}
$$

where by (58) $s_{0}=\min \left\{s=1,2 \ldots \max \operatorname{supp}\left(w_{s-1}\right)<\varepsilon_{n_{1}} \sqrt{f(k)}\right\}$. It follows for $s>s_{0}$ from (80) that

$$
\sqrt{f(k)}<\frac{\max \operatorname{supp}\left(z_{s_{0}}\right)}{\varepsilon_{n_{1}}} \leq \sqrt{f\left(\varepsilon_{n_{s}} j_{n_{s}}\right)}
$$

and thus, that $k / j_{q_{n_{s}}}<\varepsilon_{n_{s}} \leq \varepsilon_{n_{1}}$ which yields,

$$
\left|z^{*}(y)\right| \leq \frac{\left|z_{t_{0}}^{*}(y)\right|}{\sqrt{f(k)}}+5 \frac{\max _{s \leq m}\left|a_{s}\right|}{\sqrt{f(k)}}
$$

and allows us to conclude from Proposition 5.1 that the spreading model of $\left(w_{n}\right)$ is equivalent to the unit vector basis in $S$.

Together with (86) we therefore proved the following result.

Theorem 6.1. There is a constant $c>1$ so that in every block subspace of $\mathcal{G M}$ we can find block sequences $u_{n}$ and $v_{n}$, with $u_{1}<v_{1}<u_{2}<v_{2}<\ldots$, so that $\left(u_{n}-v_{n}\right)$ has a spreading model which is c-equivalent to the unit vector basis of $S$, and the sequences $\left(u_{n}\right),\left(v_{n}\right)\left(u_{n}+v_{n}\right)$ have spreading models which $c^{-1}$-dominate the norm $\|\cdot\|_{f^{1 / 2}}$ which was introduced in Section 圆. I.e. if we put $x_{n}=u_{n}, v_{n}$ or $u_{n}+v_{n}$, for $n \in \mathbb{N}$, and we denote by $\left(E,\|\cdot\|_{E}\right)$ the spreading model of $\left(x_{n}\right)$ and its basis by $\left(e_{j}\right)$ then

$$
c\left\|\sum_{s=1}^{\infty} a_{s} e_{s}\right\|_{E} \geq\left\|\left(a_{s}\right)\right\|_{f^{1 / 2}}=\max _{m \in \mathbb{N}, s_{1}<s_{2}, \ldots s_{m}} \frac{1}{\sqrt{f(m)}} \sum_{i=1}^{m}\left|a_{s_{i}}\right| \text { for }\left(a_{s}\right) \in c_{00} .
$$

Thus, Corollary 2.6 and Lemma 2.5 yield our final result:

Theorem 6.2. Let $\left(u_{n}\right)$ and $\left(v_{n}\right)$ be as in Theorem 6.1. Then there is a subsequence $\left(n_{k}\right)$ of $\mathbb{N}$ so that $\left(u_{n_{k}}\right)$ and $\left(v_{n_{k}}\right)$ are equivalent.

Proof. Using Corollary 2.6 and Lemma 2.3 twice, we may assume that

$S_{1}:\left[u_{n}: n \in \mathbb{N}\right] \rightarrow\left[u_{n}-v_{n}: n \in \mathbb{N}\right], \quad$ defined by $u_{n} \mapsto u_{n}-v_{n}$, and

$S_{2}:\left[v_{n}: n \in \mathbb{N}\right] \rightarrow\left[u_{n}-v_{n}: n \in \mathbb{N}\right]$, defined by $v_{n} \mapsto u_{n}-v_{n}$, for $n \in \mathbb{N}$

are bounded. So the bounded map $I d_{\mid\left[u_{n}\right]}-S_{1}$ defines $u_{n} \mapsto v_{n}$, while $I d_{\mid\left[v_{n}\right]}+S_{2}$ defines $v_{n} \mapsto u_{n}$, proving the claim. 
Remark. It is worth noting that when $\left(u_{n}\right)$ and $\left(v_{n}\right)$ are intertwined and equivalent in $\mathcal{G M}$, the sequences $\left(v_{n}\right)$ and $\left(u_{n+1}\right)$ are not, in general, equivalent. Otherwise the shift on $\left[u_{n}: n \in \mathbb{N}\right]$ would be an isomorphism and we would obtain an isomorphism of a subspace of $\mathcal{G} \mathcal{M}$ with its hyperplanes. But this is impossible if $\left(u_{n}\right)$ was picked inside an HI subspace of $\mathcal{G M}$.

\section{Consequences of the main Result}

\subsection{Asymptotic unconditionality.}

Recall that a seminormalized basis $\left(e_{n}\right)$ is said to be asymptotically unconditional if there exists a constant $C \geq 1$ such that for any $k \in \mathbb{N}$ and any successive blocks $k<x_{1}<\cdots<x_{k}$ on the basis, the sequence $\left(x_{1}, \ldots, x_{k}\right)$ is $C$-unconditional. The following is an easy consequence of Theorem 6.1.

Proposition 7.1. The space $\mathcal{G} \mathcal{M}$ does not contain any asymptotically unconditional block sequence.

We recall that the asymptotically unconditional HI space $G$ of Gowers is tight by range [FR2] and therefore contains no intertwined and equivalent block sequences.

The sequences $\left(u_{n}\right)$ and $\left(v_{n}\right)$ are chosen in an arbitrary, but fixed subspace $Y$ of $\mathcal{G M}$, and this is why our techniques do not seem to imply that $\mathcal{G} \mathcal{M}$ is HI (although we suspect it is). This restriction is essentially technical, however, since as we shall now see, by using Gowers' Ramsey theorem, it disappears when passing to an appropriate subspace of $\mathcal{G M}$.

\subsection{Applications of Gowers' Theorem.}

Recall that Gowers' game $\mathcal{G}_{X}$ in a space $X$ with a basis is a game between two players, where Player 1 plays block subspaces $Y_{n}$ of $X$ and Player 2 successive blocks $y_{n} \in Y_{n}$, the outcome of the game being the block-sequence $\left(y_{n}\right)$.

The set $b(X)$ of block-sequences of $X$ is seen as a subset of $X^{\omega}$ equipped with the product of the norm topology on $X$. Also for $\Delta=\left(\delta_{n}\right)_{n}$ a sequence of positive number, and $\mathbb{A} \subset b(X)$, the set $\mathbb{A}_{\Delta}$ is defined as

$$
\mathbb{A}_{\Delta}=\left\{\left(x_{n}\right) \in b(X) \mid \exists\left(y_{n}\right) \in \mathbb{A},\left\|y_{n}-x_{n}\right\| \leq \delta_{n} \forall n\right\} .
$$

Theorem 7.2 (Gowers' Ramsey Theorem, G4]). Let $X$ be a space with a basis, and $\mathbb{A}$ an analytic subset of $b(X)$. Let $\Delta>0$. Then there exists a block-subspace $Y$ of $X$ such that $\mathbb{A} \cap b(Y)=\emptyset$, or such that Player 2 has a winning strategy in Gowers' game $\mathcal{G}_{Y}$ to produce an outcome in $\mathbb{A}_{\Delta}$.

Given $c \geq 1$, consider the set $\mathbb{A}$ of block sequences $\left(x_{n}\right)_{n}$ in $\mathcal{G} \mathcal{M}$ such that $\left(x_{2 n}-\right.$ $x_{2 n+1}$ ) has a spreading model which is $c$-equivalent to the unit vector basis of $S$, and the sequences $\left(x_{2 n}\right),\left(x_{2 n+1}\right)\left(x_{2 n}+x_{2 n+1}\right)$ have a spreading model which $c^{-1}$ dominate the norm $\|\cdot\|_{f^{1 / 2}}$. It is easily checked that $\mathbb{A}$ is Borel. So up to modifying the constant $c$ to take into account a small enough perturbation $\Delta$, we may apply Gowers' Theorem to find a block-subspace $Y$ of $\mathcal{G M}$, so that the vectors $u_{n}$ and $v_{n}$ of Theorem 6.1 may be chosen in arbitrary block-subspaces of $Y$ prescribed by Player 1 . 
Proposition 7.3. There exists $c \geq 1$ and a block subspace of $\mathcal{G} \mathcal{M}$ in which Player 2 has a winning strategy to produce $u_{1}<v_{1}<u_{2}<v_{2}<\ldots$, so that $\left(u_{n}-v_{n}\right)$ has a spreading model which is c-equivalent to the unit vector basis of $S$, and the sequences $\left(u_{n}\right),\left(v_{n}\right)\left(u_{n}+v_{n}\right)$ have a spreading models which $c^{-1}$ dominate the norm $\|\cdot\|_{f^{1 / 2}}$.

Note that $\left\|\sum_{i=1}^{m} e_{i}\right\|_{f^{1 / 2}}=m f(m)^{-1 / 2}$ while $\left\|\sum_{i=1}^{m} e_{i}\right\|_{S}=m f(m)^{-1}$. So for any $\epsilon>0$, one can find $m \in \mathbb{N}$ with following property: for any $U, V$ block subspaces of $Y$, there exist $u_{1}<v_{1}<\cdots<u_{m}<v_{m}$, with $u_{i} \in U, v_{i} \in V$ for each $i$, such that $\left\|\sum_{i=1}^{m} u_{i}-v_{i}\right\|<\epsilon\left\|\sum_{i=1}^{m} u_{i}+v_{i}\right\|$, which of course implies the HI property. This property is actually the uniform version of the HI property which appears as the counterpart of asymptotic unconditionality in the dichotomy proved by Wagner [W].

The third dichotomy implies that we may assume that the space of Proposition 7.3 is tight, and the fourth dichotomy that it is subsequentially minimal. Actually slightly more may be observed.

Theorem 7.4. There exists a tight $H I$ block-subspace $\mathcal{X}_{G M}$ of $\mathcal{G} \mathcal{M}$ with a normalized basis which is subsequentially minimal. More precisely, there exists $c \geq 1$, such that for any block-subspace $Y$ of $\mathcal{X}_{G M}$, there exists a block-sequence $\left(y_{k}\right)$ of $Y$ and a subsequence $\left(f_{k}\right)$ of the basis of $\mathcal{X}_{G M}$ such that

(a) $y_{1}<f_{1}<y_{2}<f_{2}<\cdots$

(b) $\left(y_{k}\right),\left(f_{k}\right),\left(y_{k}+f_{k}\right)$ have spreading models which $c^{-1}$ dominate the norm $\|\cdot\|_{f^{1 / 2}}$,

(c) $\left(y_{k}-f_{k}\right)$ has a spreading model which is c-equivalent to the unit vector basis of $S$,

(d) consequently, $\left(f_{k}\right)$ is equivalent to $\left(y_{k}\right)$.

This is a variation on [FR1, Proposition 6.5]. Since the proof is much shorter than the demonstration of the fourth dichotomy, we give a sketch of it.

Proof. Let $\mathbb{A} \subset b(\mathcal{G M})$ be defined as after Theorem 7.2. Using Gowers' first dichotomy (see Theorem [1.1), the fact that no HI space has a minimal subspace, and the third dichotomy proven in [FR1] (see Theorem 1.3) we may pass to an HI tight subspace. By Theorem 6.2 and Gowers' Ramsey Theorem (Theorem 7.2) we can, after modifying $c$, assume that Player 2 has a winning strategy in Gowers' game to play inside $\mathbb{A}$; also we may and shall only use blocks with rational coordinates in this proof (and assume Gowers' game is played with such blocks). Then the finite block-sequences of initial moves prescribed by the winning strategy of Player 2 form a non-empty tree $T$ which does not have any maximal elements. We denote by $[T]$ the infinite block sequences $\left(x_{j}\right)$ for which all the initial segments $\left(x_{j}\right)_{j=1}^{n}, n \in \mathbb{N}$, lie in $T$. Then $[T] \subseteq \mathbb{A}$ and for all $\left(y_{0}, \ldots, y_{m}\right) \in T$ and all block sequences $\left(z_{n}\right)$, there is a block $y_{m+1}$ of $\left(z_{n}\right)$ such that $\left(y_{0}, \ldots, y_{m}, y_{m+1}\right) \in T$, [FR1, Lemma 6.4]. Since $T$ is countable, we can construct inductively a block sequence $\left(v_{n}\right)$ such that for all $\left(u_{0}, \ldots, u_{m}\right) \in T$ there is some $v_{n}$ with $\left(u_{0}, \ldots, u_{m}, v_{n}\right) \in T$.

We claim that $\mathcal{X}_{G M}:=\left[v_{n}, n \in \mathbb{N}\right]$ works. Indeed if $\left(z_{n}\right)$ is any block sequence of $\left(v_{n}\right)$, we may construct inductively a block-sequence $\left(y_{i}\right)$ of $\left(z_{n}\right)$ and a subsequence $\left(f_{i}\right)$ of $\left(v_{n}\right)$ such that $\left(y_{0}, f_{0}, \cdots, y_{n}, f_{n}\right)$ belongs to $T$ for all $n$. Therefore $\left(y_{0}, f_{0}, y_{1}, f_{1}, \cdots\right)$ 
belongs to $\mathbb{A}$. Finally the normalized basis $\left(v_{n} /\left\|v_{n}\right\|\right)$ of $\mathcal{X}_{G M}$ satisfies the conclusion of Theorem 7.4 .

Since this construction can be done in any block-subspace of $\mathcal{G} \mathcal{M}$, we may assume that $\mathcal{X}_{G M}$ is actually sequentially minimal.

\subsection{Local minimality.}

We briefly expose the fifth dichotomy obtained in [FR1, which is related to the second general kind of tightness called tightness with constants. A space $X=\left[e_{n}\right]$ is tight with constants when for for every infinite dimensional space $Y$, the sequence of successive subsets $I_{0}<I_{1}<\ldots$ of $\mathbb{N}$ witnessing the tightness of $Y$ in $X$ may be

chosen so that $Y \nsubseteq_{K}\left[e_{n} \mid n \in \mathbb{N} \backslash I_{K}\right]$ for each $K$. Equivalent no infinite dimensional space embeds uniformly into the tail subspaces of $X$ [FR1, Proposition 4.1]. This is the case for Tsirelson's space $T$ or its $p$-convexified version $T^{(p)}$.

On the other hand we already mentioned that a space $X$ is said to be locally minimal if there exists a constant $K \geq 1$ such that every finite dimensional subspace of $X K$-embeds into every infinite dimensional subspace of $X$.

Theorem 7.5 (Fifth dichotomy [FR1]). Any Banach space contains a subspace with a basis which is either tight with constants or locally minimal.

Since $S$ contains $\ell_{\infty}^{n}$ 's uniformly and since $\mathcal{G M}$ is saturated with sequences with spreading model $c$-equivalent to the basis of $S, \mathcal{G M}$ also contains $\ell_{\infty}^{n}$ 's uniformly in every subspace. So by the universal properties of these spaces, $\mathcal{G M}$ is locally minimal.

Theorem 7.6. There exists a locally and sequentially minimal HI Banach space.

Since an HI space does not contain a minimal subspace, this answers [FR2, Problem 5.2], that is, the space $\mathcal{G} \mathcal{M}$ demonstrates that there are other forms of tightness than tightness by range or with constants.

The fifth dichotomy and a dichotomy due to A. Tcaciuc [Tc] are used in [FR1] to refine the types (1)-(6) into subclasses. In their terminology, $\mathcal{X}_{G M}$ is of type (2b).

\subsection{Open problems.}

The most important problem which remains open in Gowers' classification program is whether there exist spaces of type (4). Note that such a space would satisfy the criterion of Casazza, and therefore would not be isomorphic to its proper subspaces.

Problem 7.7. Find a space with an unconditional basis, tight by range and quasiminimal.

The nature of the tightness of $\mathcal{X}_{G M}$ remains to be understood. This property is a consequence of the non-minimality of HI spaces and of the third dichotomy, with no information on how the sequence $\left(I_{n}\right)$ of subsets of $\mathbb{N}$ depends on the subspace $Y$.

Problem 7.8. Find information on the sequences $\left(I_{n}\right)$ in the definition of the tightness of $\mathcal{X}_{G M}$. Is $\mathcal{G M}$ or $G M$ itself tight? 
C. Rosendal $\left[\mathrm{R}\right.$ defined notions of $\alpha$-minimality and $\alpha$-tightness, where $\alpha<\omega_{1}$ is an ordinal. Local minimality implies that $\mathcal{X}_{G M}$ is $\omega^{2}$-minimal and not $\omega$-tight. On the other hand, being tight, it must be $\alpha$-tight for some $\alpha<\omega_{1},[\underline{\mathrm{R}}$, Theorem 3].

Problem 7.9. Find $\min \left\{\alpha \in \omega_{1} \mid \mathcal{X}_{G M}\right.$ is $\alpha$-tight $\}$.

It is unknown whether an HI space may be tight with constants. With the exception of the uniformly convex HI space of [F1], examples of the Gowers-Maurey family usually contain $\ell_{\infty}^{n}$ 's uniformly - and therefore are locally minimal.

Problem 7.10. Find an HI space which is tight with constants.

\section{REFERENCES}

[AS] G. Androulakis and Th. Schlumprecht, Strictly singular, non compact operators exist on the space of Gowers and Maurey, Jour. London Math. Soc. 64 (2001) 655 - 674.

[AS2] G. Androulakis and Th. Schlumprecht, The Banach space $S$ is complementably minimal and subsequentially prime. Studia Math. 156 (2003), no. 3, 22 - 242.

[C] P.G. Casazza, Handwritten notes.

[DOSZ] S. Dilworth, E. Odell, Th. Schlumprecht, and A. Zsák, Partial unconditionality. Houston J. Math. 35 (2009), no. 4, 1251 - 1311.

[El] J. Elton, Weakly null normalized sequences in Banach spaces. Ph.D. thesis, Yale Univ., 1978.

[F1] V. Ferenczi, A uniformly convex hereditarily indecomposable Banach space, Israel Journal of Mathematics 102 (1997), 199-225.

[F2] V. Ferenczi, Operators on subspaces of hereditarily indecomposable Banach spaces, Bull. London Math. Soc. 29 (1997), 338-344.

[FG] V. Ferenczi and G. Godefroy, Tightness of Banach spaces and Baire category, preprint.

[FR1] V. Ferenczi and C. Rosendal, Banach spaces without minimal subspaces, Journal of Functional Analysis 257 (2009) 149 - 193.

[FR2] V. Ferenczi and C. Rosendal, Banach spaces without minimal subspaces - examples, Annales de l'Institut Fourier, to appear.

[G1] W.T. Gowers, A solution to Banach's hyperplane problem, Bull. London Math. Soc. 26 (1994), no. 6, 523-530.

[G2] W.T. Gowers, A hereditarily indecomposable space with an asymptotic unconditional basis, Geometric aspects of functional analysis (Israel, 1992-1994), 112-120, Oper. Theory Adv. Appl., 77, Birkhaüser, Basel, 1995.

[G3] W.T. Gowers, A new dichotomy for Banach spaces, Geom. Funct. Anal. 6 (1996), no. 6, 1083-1093.

[G4] W. T. Gowers, An infinite Ramsey theorem and some Banach-space dichotomies. Ann. of Math. 2156 (2002), no. 3, $797-833$.

[GM] T. Gowers and B. Maurey, The unconditional basis problem, Jour. Am. Math. Soc. 6, no. 4, 851- 874 .

[KL] D. Kutzarova and P.-K. Lin, Remarks about Schlumprecht space. Proc. Amer. Math. Soc. 128 (2000), no. 7, $2059-2068$.

[MP] A. Manoussakis and A. Pelczar, Quasi-minimality in mixed Tsirelson's spaces, Math. Nachrichten, to appear.

[Od] E. Odell, On Schreier unconditional sequences. Banach spaces (Mérida, 1992), 197-201, Contemp. Math., 144, Amer. Math. Soc., Providence, RI, 1993.

[R] C. Rosendal, $\alpha$-minimal Banach spaces, preprint.

[Sch1] Th. Schlumprecht, An arbitrarily distortable Banach space, Israel J. Math. 76(1991), no. 1-2, 81-95. 
[Sch2] Th. Schlumprecht, How many operators exist on a Banach space? Trends in Banach spaces and operator theory (Memphis, TN, 2001), 295 - 333, Contemp. Math., 321, Amer. Math. Soc., Providence, RI, 2003.

[Tc] A. Tcaciuc, On the existence of asymptotic- $\ell_{p}$ structures in Banach spaces, Canad. Math. Bull. 50 (2007), no. 4, 619-631.

[Ts] B.S. Tsirelson, Not every Banach space contains $\ell_{p}$ or $c_{0}$, Functional Anal. Appl. 8 (1974), 138-141.

[W] R. Wagner, Finite high-order games and an inductive approach towards Gowers's dichotomy, Proceedings of the International Conference "Analyse \& Logique" (Mons, 1997). Ann. Pure Appl. Logic 111 (2001), no. 1-2, 39-60.

Address of $V$. Ferenczi:

Departamento de Matemática, Instituto de Matemática e Estatística, Universidade de São Paulo, rua do Matão, 1010, 05508-090 São Paulo, SP, Brazil.

ferenczi@ime.usp.br

Address of Th. Schlumprecht: Department of Mathematics, Texas A\&M University, College Station, Texas, 77843, USA schlump@math.tamu .edu 